Review

\title{
Recent advances in smart hydrogels for biomedical applications: From self- assembly to functional approaches
}

\author{
N.N. Ferreira, L.M.B. Ferreira, V.M.O. Cardoso, F.I. Boni, A.L.R. Souza, M.P.D. Gremião* \\ School of Pharmaceutical Science, São Paulo State University, UNESP, Rodovia Araraquara Jaú, Km 1, Araraquara, São Paulo, Brazil
}

\section{A R T I C L E I N F O}

\section{Keywords:}

Smart hydrogels

Functional approach

Drug delivery systems

Dynamic interactions

Functional biomaterials

Responsiveness

\begin{abstract}
A B S T R A C T
This review discusses basic aspects used to control the architecture and functional properties of smart hydrogels. The introduction briefly outlines what has been accomplished regarding smart hydrogels and explores historical aspects and the fundamental understanding of these systems. Then, a short discussion on the chemical interactions and the main variables involved in architectural construction is exhibited. Further analysis provides the basis for optimizing biological responses through system modulation. Finally, we outline perspectives and challenges for building smart hydrogels into functionalized and modulated delivery systems.
\end{abstract}

\section{Introduction}

The development of hydrogels as functional biomaterials has revolutionized the field of study concerning responsive materials. Initially, hydrogels were developed for biomedical applications, especially tissue scaffolds and contact lenses [1]. In the late 19th century, hydrogels were first described as colloidal gels from inorganic salt. Around the middle of the 20th century, Wichterle and Lim termed them as water-swollen crosslinked polymeric network(s) [2,3]. Currently, hydrogels can be defined as three-dimensional networks of hydrophilic polymers. These systems have the ability to swell and absorb large amounts of water or biological fluids without losing their structure [4-6]. Their capacity to absorb liquid is due the presence of hydrophilic compounds attached to the polymer chains, such as amid, amino, carboxyl and hydroxyl groups, which are able to ionize in the presence of water. Furthermore, their physical properties, such as swelling, surface characteristics and mechanical strength, can be modulated by physicochemical reactions to improve elasticity and mechanical resistance, which are important features to considered when developing delivery systems $[3,7,8]$.

The most common terms associated with hydrogels are: ocular lens, wound healing [9], super-absorbents [10], tissue engineering, tissue scaffolds, cell immobilization [11] and drug delivery systems [12]. The number of publications reported in the Science Direct database involving hydrogels, containing such keywords, over the last ten years showed exponential growth, including books, journal articles and reference works (Fig. 1). Although this popularity can be observed for all aforementioned subjects, tissue engineering and drug delivery standout since they represent the majority of the publications. Remarkable, nonetheless, is the similar increase of studies regarding tissue scaffolds, tissue engineering, wound healing and drug delivery.

Since 2000, the number of publications involving the term 'hydrogels' expanded notably [13]. In parallel, the wide applicability of hydrogel as functional materials has attracted great interest among researchers and several industry segments [14].

Unlike conventional hydrogels, which exhibit swelling/deswelling properties linked to water availability, smart or stimulus-responsive hydrogels are the most promising materials because of their additional properties. These types of hydrogels are most often related to the physical, chemical and biological fields $[13,15,16]$ and their unique properties can be associated with environmental factors, such as external stimuli that promotes a change in organization.

Although the first studies related to hydrogels appeared in 1894, the word "smart" was introduced in 1948 by Kuhn and co-workers [17]. This most recent nomenclature gained importance because they are capable of exploitation based on specific triggers (stimuli) to induce changes in structure and function [3]. The first publication reported was on poly(acrylic acid) polymer molecules that could undergo structural adjustments according to the media $\mathrm{pH}$ [18]. At the same time, publications reported such profiles for different polymeric networks including the pioneering smart hydrogels [18-21]. This drug delivery concept is denominated as "smart" because it can detect prevailing stimuli and respond through structural, morphological or functional changes resulting in the release of entrapped drugs in a controlled manner [22].

A set of criteria has been used to classify these systems, including

\footnotetext{
* Corresponding author at: Faculdade de Ciências Farmacêuticas, UNESP, Rodovia Araraquara Jaú, Km 01 - s/n, Araraquara, SP 14800-903, Brazil.

E-mail address: pgremiao@fcfar.unesp.br (M.P.D. Gremião).
} 


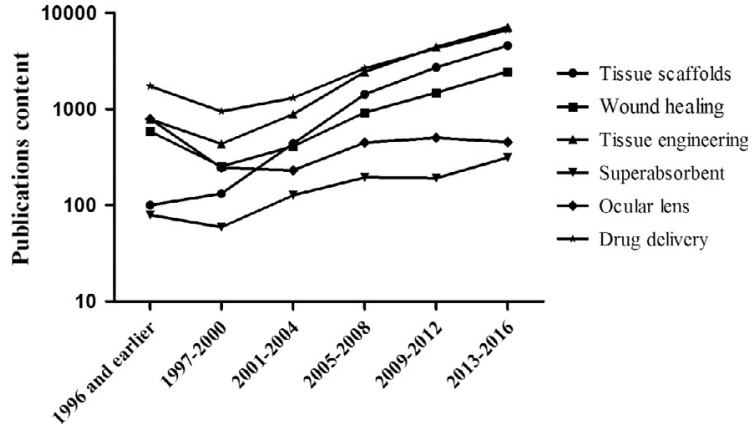

Fig. 1. Summary of the content found related to common terms in the Science Direct database. Publications include journal articles, books and reference works. Research conducted in September 2017

origin (natural or synthetic), degradability and cross-linking mechanisms for self-assembly. Chemically cross-linked hydrogel networks are generated by covalent bounds between polymer chains resulting in permanent junctions [3]. On the other hand, physically cross-linked structures are designed through supramolecular forces (noncovalent) forming rapid and reversible networks, a feature that we are particularly interested in [2]. Smart hydrogels can be classified by several criteria. Publications focused on drug delivery have primarily considered external types of stimuli. Responses can be induced by physical or chemical stimuli. The first type includes: pressure, light, temperature, and magnetic or electric fields. Chemical or biochemical stimuli include ionic strength, $\mathrm{pH}$ and ions. Complementing these factors, punctual molecular events, chemical or biological species availability can also be explored $[7,8,13]$. Moreover, these systems can be designed to respond a multiple stimuli listed above [21].

Many reviews have been reported to introduce the field of stimuli responsive hydrogel systems, although a large portion of these publications focused only on applications. The present review focuses on the basic concepts and responsivity mechanisms guided by dynamic interactions that provide valuable tools for designing smart hydrogels for functional approaches.

\section{Self-assembly for designing smart hydrogels}

Supramolecular chemistry is defined as the chemistry beyond the molecule. This field has evolved by engaging the fundamental and applied aspects of supramolecular interaction manipulation [23,24]. Although a major focus has been on physical interactions (e.g., hydrophobic, hydrogen bond and electrostatics), the dynamic nature of supramolecular chemistry makes it applicable to the development of smart hydrogels. In this sense, not only non-covalent links but also dynamic covalent bonds can be explored in tailoring stimuli-responsiveness [25-27]. Smart hydrogels might self-assemble by establishing supramolecular interactions such as ionic bonds, weak physical entanglements, and hydrogen bonds. However, covalent chemical links may also be used to obtain the same results [28]. The self-assembly of highly organized macromolecular systems nanostructured by dynamic interactions has been strongly explored in drug delivery systems [2].

Their dynamic nature is an essential feature for the practical design of responsive systems. Therefore, it is mandatory to understand the concept of the dynamics in order to associate its meaning with hydrogels categorized as "smart". The static behavior of conventional hydrogels versus the dynamic behavior of smart hydrogels are two extremes of behavior that can significantly affect the performance of drug delivery systems (Fig. 2). This paradigm shift that contrasts the static and dynamic nature of materials has been clearly identified in hydrogel designs. Thus, traditional static hydrogels designed as bioinert materials have been replaced by dynamic systems that incorporate sophisticated functions $[29,30]$.

The physicochemistry behind the supramolecular interactions and the dynamic covalent bonds are of primary importance to the synthesis steps and performance of biological systems. By manipulating the factors involved in these links, we control the structural properties of the final system. In this context, many supramolecular and dynamic covalent bonds have been explored through the advent of smart hydrogels, as summarized in Table 1.

The development of hydrogels based on supramolecular and dynamic covalent chemistry enables the biological functions that are impossible to achieve by static links. The modulation of drug release from delivery systems, self-healing and tissue adhesiveness are some of the interesting functions that can be tailored by controlling the system properties during the synthesis procedure $[39,40]$. The dynamics of smart hydrogels can be designed to respond to a physical, chemical or biological stimulus. Upon receiving these external inputs, the hydrogel undergoes a structural/morphological property change, which leads to the desired outcomes. This off-on transition is ultimately responsible for achieving the biological function. Expected responses can be manifold and include degradation, drug release, swelling, changes in shape or surface, conformational modifications or micellization [41].

The final morphology expected for the hydrogel is associated with a series of variables, which primarily includes the synthesis or treatment procedures of the original polymer, monomer composition and their ratios, and the crosslinking method [42].

Polymer science and drug delivery together represent most of the active branches in the development of therapeutics [43]. In drug delivery systems, pharmaceutically active compounds are loaded into a hydrogel, which is then administered to the body where the drugs are released in a sustained manner for periods ranging from several days to several months. Furthermore, introduction or association of the drug to the polymeric pattern can provide a passive function as a drug carrier, alter the degradation process, or minimize immunogenicity and toxicity, in addition to possibly increasing circulation time [41]. Therefore, the frequency of drug administration can be decreased and peak concentrations can be avoided, reducing potentially harmful side effects. Alternatively, hydrogels are used specifically to deliver its content to the target area. Therefore, the hydrogel structure might protect drugs from hostile environments and control drug release by changing the gel structure in response to environmental stimuli.

To further reflect on these systems, the following section will outline different classes of smart hydrogels and their specific features, including functional groups and factors involved in responsiveness. Furthermore, selected comprehensible examples in drug delivery will be given, and, to conclude, a brief outlook of future perspectives will be addressed.

\subsection{Thermo-responsive hydrogels}

Thermo-responsive hydrogels are one of the most studied classes of stimuli systems in tissue engineering and drug delivery research, as medium temperature may fluctuate in physiological and pathological conditions. These hydrogels, composed mainly of natural and synthetic polymers with balanced hydrophilic and hydrophobic groups in their chains (Table 2) are characterized by their ability to undergo reversible modifications as volume changes. They also swell or de-swell in response to a critical temperature, which causes a sudden change in the solvation of their molecules, conformational state and, consequently, water-solubility [41,44-47].

Thermo-responsive hydrogels can be divided into two groups according to their behavior with the surrounding solvent molecules. Extensively studied, lower critical solution temperature (LCST) hydrogels exhibit non-linear responses, where the polymer's solubility decreases as the temperature increases, leading to the formation of a more structures gel. The second type, upper critical solution temperature (UCST) systems, on the other hand, become soluble upon heating.

For the LCTS hydrogels, at lower temperatures, the water molecules are arranged around the polymer, establishing hydrogen-bonds with the 


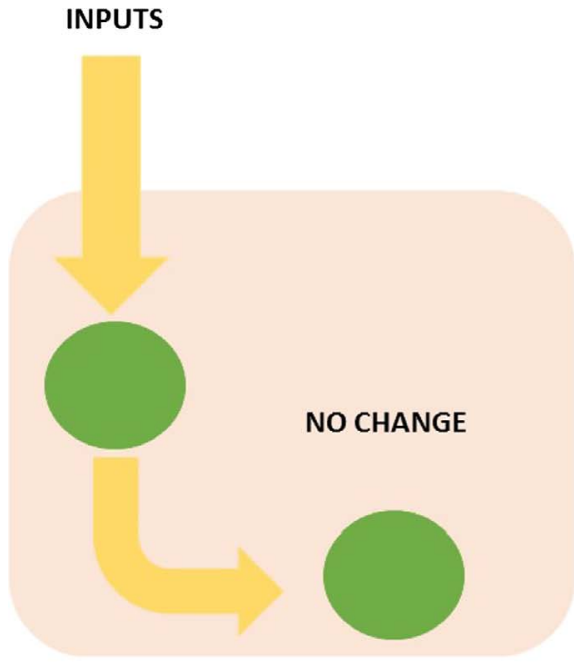

STATIC DRUG DELIVERY SYSTEM
INPUTS

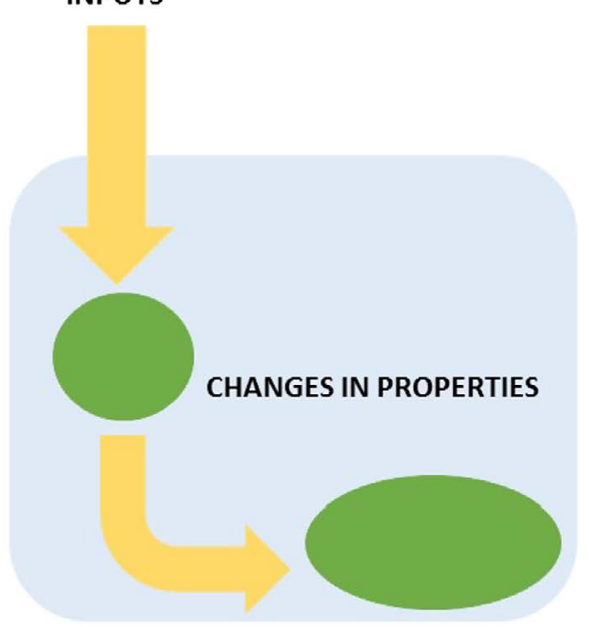

DYNAMIC DRUG DELIVERY SYSTEM
Fig. 2. Visual scheme illustrating the parallel between static and dynamic designs: two behavioral extremes that can significantly affect the performance of drug delivery systems. hydrophilic groups of the structure, resulting in a small change in enthalpy and, consequently, low entropy. However, the increase of environmental temperature reduces the energy associated to the waterpolymer interaction, allowing an increase in the energy associated to the polymer-polymer interaction. Consequently, the change in enthalpy increases. To maintain the change in Gibbs free energy low and to compensate this increase in the enthalpy term $(\Delta H)$, water molecules dissociate from the polymer and the entropy increases. Thus, the polymer dehydrates, transforming itself into a more hydrophobic structure and, lastly, causing phase separation (Fig. 3) [48].

The value of the critical temperature point usually depends on the molecular weight of the polymer, which is lower for longer polymers or for the hydrogen-bonding capabilities of constituent monomer units [49-51].

Typical synthetic polymers used to develop LCST systems are poly (N-isopropylacrylamide) (PNIPAM), poly(N,N-diethyl acrylamide) (PDEAM), poly(methylvinylether) (PMVE), poly(N-vinylcaprolactam) (PVC), copolymer blocks of poly(ethylene oxide), known as Pluronics ${ }^{\circledR}$ and Tetronic ${ }^{\oplus}$, and poly(pentapeptide) of elastin.

LCST polymers display critical temperature transitions when exposed to a wide range of temperatures, however, biomedicine is more interested in those able to respond at $30-37^{\circ} \mathrm{C}$, close to the body temperature (Table 3). PNIPAM and PDEAM have similar transition temperatures and are the most commonly used polymers in thermoresponsive hydrogel development. For PDEM, the LCST is influenced by polymer tacticity, i.e., the regularity in the arrangement of side groups. On the other hand, for PNIPAM, the transition temperature can be influenced by molecular design with changes in the hydrophilic/hydrophobic balance which is independent of the polymer concentration and molecular weight. The PNIPAM response against increases in temperature (from 32 to $35{ }^{\circ} \mathrm{C}$ ) was first described by Heskins and Guillet, generating a particular interest in drug delivery systems design, especially due to its biocompatibility compared to PDEAM [52-54].

PMVE is a water soluble LCST polymer which displays temperature transition at exactly $37^{\circ} \mathrm{C}$, making it very interesting for pharmaceutical and biomedical applications. At this temperature, the hydrogen bonds are disfavored and polymer-polymer interactions are favored, promoting the aggregation of PMVE molecules.

Dispersed PMVE can be crosslinked with electrons or $\gamma$-rays. This physically produced hydrogel behaves similarly to supramolecular systems, swelling at temperatures below and shrinking at temperatures above the LCST. These systems exhibit a discontinuous temperaturedependent swelling behavior, in which concentration might represent one of the limitations of the system [52,55].

PVC, another LCST polymer, has interesting properties to be used in medical devices and in implant development, such as water solubility, biocompatibility, high swelling, transition temperature at $33^{\circ} \mathrm{C}$ and low cost $[52,55,56]$. Copolymer blocks of poly(ethylene oxide) and poly (propylene oxide) sequences are a class of commercial polymers marketed as Pluronics ${ }^{\circledast}$, or Poloxamer, and Tetronics ${ }^{\circledast}$. These polymers exhibit transition behavior when temperatures are close to the physiological temperature $\left(37^{\circ} \mathrm{C}\right)$, and, because of this, they are extensively used as drug delivery systems and injectable devices for tissue engineering processes. Pluronics ${ }^{\circledR}$ and Tetronics $^{\circledR}$ have an amphiphilic character, and gelation occurs by 3-dimensional packing of the polymer molecules in micelles due to hydrophilic-hydrophobic balance. The LCSTs of hydrogels composed by Poloxamer ${ }^{\circledR}$ and Tetronics ${ }^{\circledR}$ are mainly dependent on their concentration and can be modulated by incorporating different side chains with hydrophilic or hydrophobic segments [44,52]. These synthetic polymers are very useful, but one of their limitations is their non-biodegradability and, for some applications as drug delivery systems, this feature is indispensable.

Despite presenting higher transition temperatures, some natural polymers such as cellulose derivatives, chitosan and gellan gum hold a good biodegradability and can be used to develop thermo-responsive hydrogels. After water dispersion and heating, most of these natural polymers form a gel phase when the temperature is lowered. Cellulose derivatives such as methylcellulose and hydroxypropyl methylcellulose (HPMC) are soluble in water at low temperatures, but after heating at $40{ }^{\circ} \mathrm{C}$ and $70^{\circ} \mathrm{C}$, respectively, they form opaque gels (Table 3). The gelation of these polymers is primarily caused by hydrophobic interactions between molecules containing methoxy substitutes. At low temperatures, chains are solvated, and polymer-polymer interactions are disfavored. However, when the temperature is raised, polymer molecules lose their hydrating water, which results in a strong interaction between polymers chains and, consequently, increased viscosity. For physiological applications, these transition temperatures are not applicable, but some strategies can be used to decrease the LCST. Incorporating $\mathrm{NaCl}$ decreases the methylcellulose transition temperature to $32-34^{\circ} \mathrm{C}$, and the molar substitution of HPMCP can lower the LCST to $40^{\circ} \mathrm{C}[49,57-59]$.

Chitosan (CH), a chitin deacetylated derivative, is a natural cationic biocompatible biodegradable and low-cost polymer composed of Dglucosamine and N-acetyl-D-glucosamine linked by beta $(1,4)$-glycosidic bonds. $\mathrm{CH}$ is soluble in solvents with $\mathrm{pH}$ values below 6.0 , and because of this, the gelation of $\mathrm{CH}$ solutions is $\mathrm{pH}$ dependent. However, $\mathrm{CH}$ 
polymer dispersion can become thermo-responsive by adding a polyol salt to the solution. In this case, at body temperature, $\mathrm{CH}$ chains lose their hydrating water and the polyol salt facilitates the interaction between side groups, such as hydrogen bonding, electrostatic interactions and hydrophobic interactions. Thus, $\mathrm{CH}$ hydrogels have become valuable tools for cartilage repair [49].

Gellan gum (GG) is a hydrophilic and anionic exopolysaccharide composed of repeated units of glucose, glucuronic acid, rhamnose (at a 2:1:1 molecular ratio) and two acetyl substituents, acetate and glycerate, which are linked to glucose residue adjacent to the glucuronic acid. GG is biocompatible, biodegradable and can originate hydrogels at low concentrations. Upon heating, these polymer solutions adopt a random coil conformation. Then, when temperature decreases, the chains adopt a double-helical conformation with ordered junction zones, resulting in a three-dimensional network. These characteristics make GG an interesting material for pharmaceutical applications [49,60-64].

Hydrogels composed only of natural polymers have limited responses at physiological temperature. On the other hand, synthetic polymers show very fast responses, forming structured hydrogels. However, it is necessary to be aware that biocompatibility and good biodegradability are often essential for biological use. Therefore, the association of natural and synthetic polymers can contribute to forming material with the desired properties for specific biological application.

From 2005 to 2010, 330 publications per year included LCST, while only 44 per year were addressed UCST. The main reason for this disparity is based on the fact that most UCST polymers have a transition temperature below $25^{\circ} \mathrm{C}$, which makes them infeasible for pharmaceutical and medical product development. Moreover, the biodegradability of this polymer class has not yet been proven. The UCST group is composed of acrylamide (AAm) and acrylic acid (AAc) derivatives, such as poly-3-dimethyl(methacryloyloxyethyl) ammonium propane sulfonate (PDMAPS) and poly(3-[N-(3-methacrylamidopropyl)- $N, N$-dimethyl]ammoniopropane sulfonate (PSPP), and zwitterionic polymers. Their use as UCST is dependent on their concentration in solution and ranges from $32{ }^{\circ} \mathrm{C}$ at $0.1 \%(\mathrm{w} / \mathrm{v})$ to $15^{\circ} \mathrm{C}$ at $8 \%(\mathrm{w} / \mathrm{v})$.

Several polymers can be included in both UCST and LCST classes, e.g., poly(ethylene oxide) (PEO), which shows a loop-shaped miscibility gap with UCST > LCST. The temperature transition of PEO is influenced by ionic strength and chemical crosslinking by radiation. PEO is non-biodegradable, thus its toxicity is a concerning point $[65,66]$.

Thermo-responsive hydrogels usually exhibit a pressure sensitivity response, in which the degree of swelling is influenced by hydrostatic pressure near the LCST. PNIPAM, discussed earlier, has an increased degree of swelling under increased pressure [47]. The behaviors described for LCST and UCST systems are not restricted to aqueous environments, but only aqueous systems are of biomedical interest.

Among the many applications of thermo-responsive hydrogels, their use in tissue engineering stands out most. Tan and co-authors (2009) synthesized a PNIPAM conjugated with hyaluronic acid using a thermoradical polymerization method. They observed an LCST for this hydrogel near to $30^{\circ} \mathrm{C}$, which formed a structured and porous hydrogel able to encapsulate adipocytes and resist enzymatic activity. Based on a preliminary in vivo study, the authors demonstrated the usefulness of in situ PNIPAM-hyaluronic acid hydrogel as an injectable system for adipose tissue engineering [67]. Several applications are also highlighted by the authors, including the development of drug delivery systems. Gao and contributors (2005) developed PNIPAM nanocapsules with temperature-tunable diameters and permeability by precipitation polymerization. In this work, they were able to effectively control the release of a fluorescent compound. At the LCST $\left(32{ }^{\circ} \mathrm{C}\right)$, the nanocapsules shrunk, and the release of the compound, from its internal cavity, was inhibited [68].

PMVE, as previously described, can be used in the development of drug delivery systems that respond to body temperature. Arndt and coauthors (2001) synthetized a thermo-responsive micro-hydrogel by 
Table 2

Molecular structure of thermo-responsive polymers.

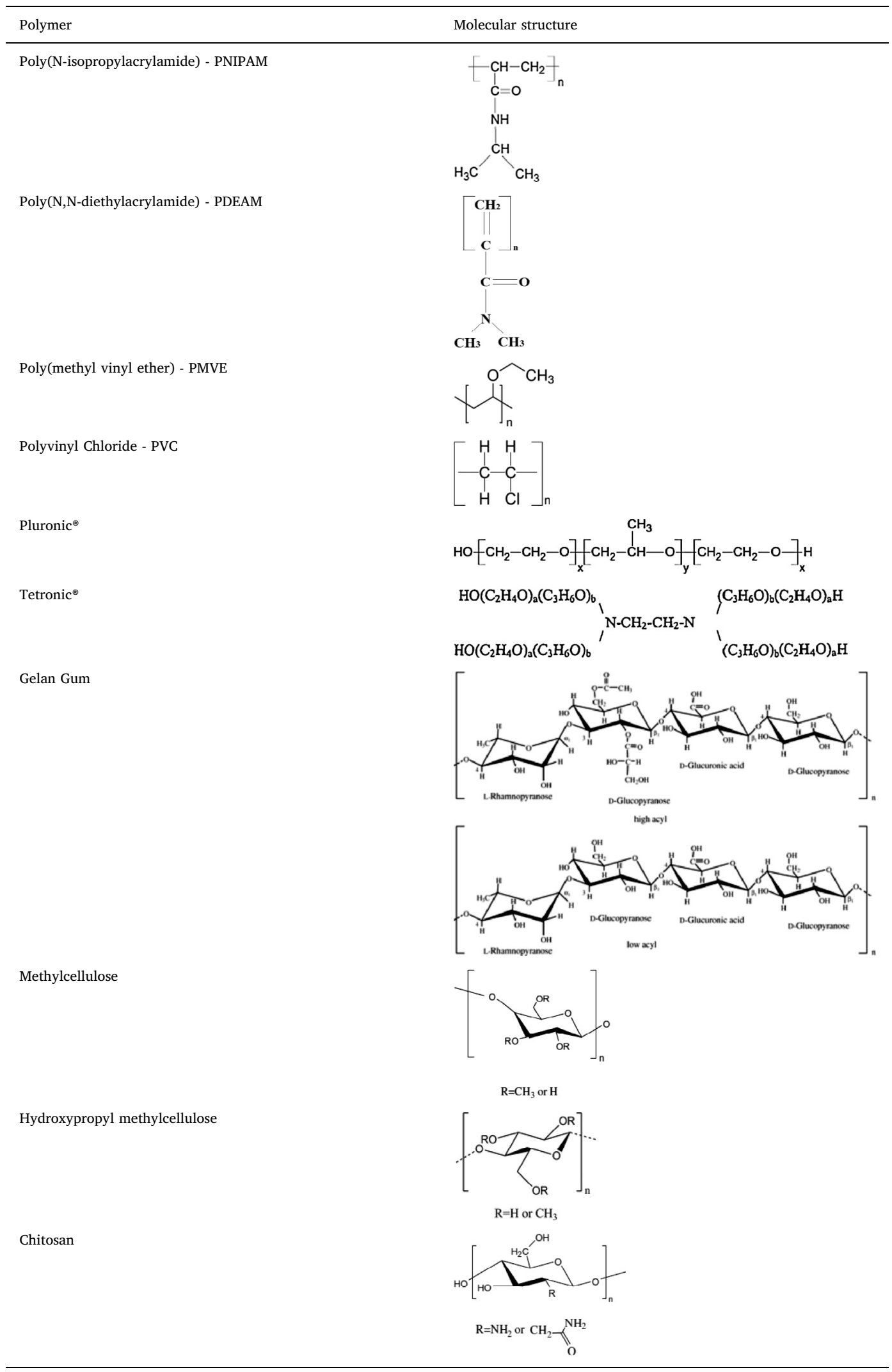



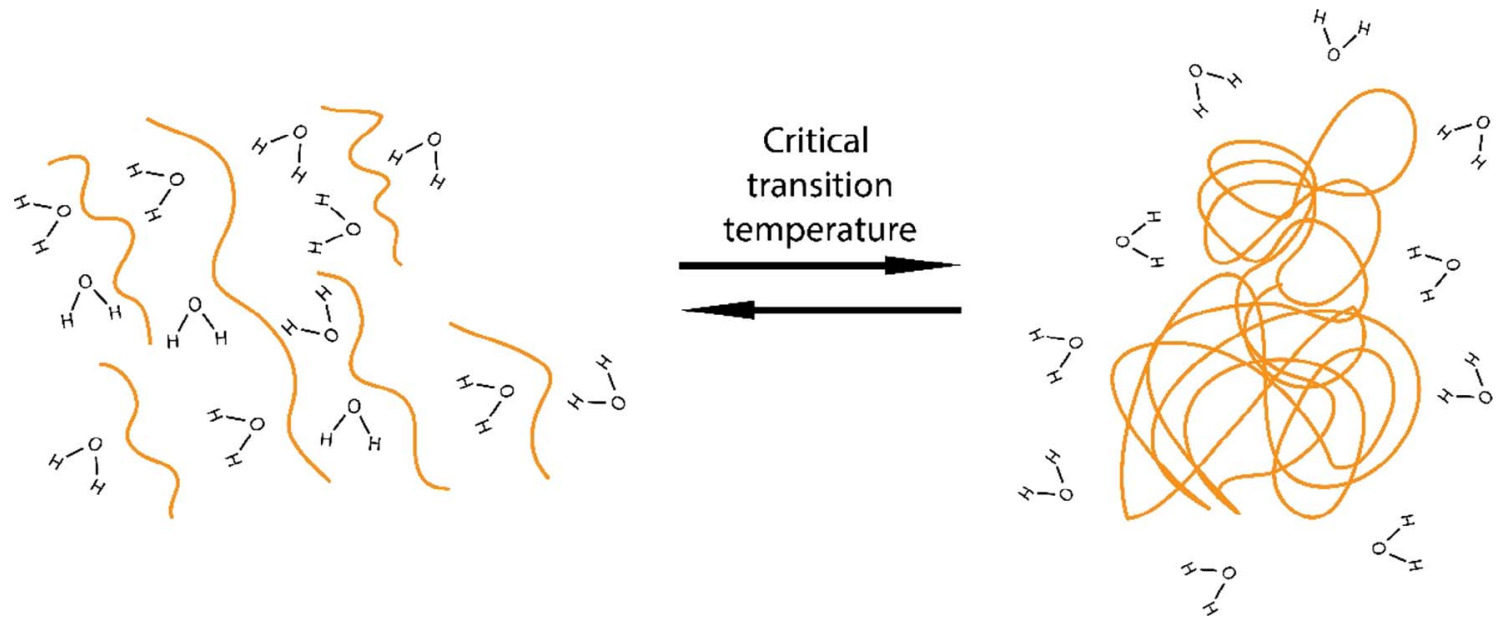

Fig. 3. Schematic illustration of LCST hydrogel behavior.

Table 3

Phase transition temperatures for polymers with LCST and UCST behavior.

\begin{tabular}{ll}
\hline Polymer & Transition temperature (in water) \\
\hline PNIPAM & $30-34{ }^{\circ} \mathrm{C}$ \\
PDEAM & $32-34^{\circ} \mathrm{C}$ \\
PMVE & $37{ }^{\circ} \mathrm{C}$ \\
PVC & $30-50{ }^{\circ} \mathrm{C}$ \\
Pluronic ${ }^{\oplus}$ and Tetronic & $30-40^{\circ} \mathrm{C}$ \\
Gellan gum & $50-60{ }^{\circ} \mathrm{C}$ \\
Methylcellulose & $40^{\circ} \mathrm{C}$ \\
HPMC & $70{ }^{\circ} \mathrm{C}$ \\
AAm and AAc & $15-25^{\circ} \mathrm{C}$ \\
\hline
\end{tabular}

using radiation. Their system consisted of microparticles that stayed in a swollen state under the LCST and rapidly shrunk near $37^{\circ} \mathrm{C}$, forming compact spheres with pores for liquid diffusion and drug release [69].

Poloxamer $^{\circledR}$ is very useful synthetic polymer with LCST, used in skin devices for drug release, for example. Pillai (2003) studied a gel formulation using poloxamer 407 for transdermal insulin delivery. The properties of this hydrogel were evaluated by ex vivo and in vivo skin permeation studies in rats with a chemical enhancer and/or iontophoresis. They demonstrated that the Poloxamer ${ }^{\circledR}$ hydrogel was physically and chemically stable during the storage period and that modulate insulin release and permeation through the skin is possible, reaching an effective plasma concentration when applying an electric current [70].

Regarding natural polymers, injectable $\mathrm{CH}$ and glycerophosphate hydrogels were described by Chenite and co-workers (2000). These authors found that, below room temperature, these formulations are liquid which permits encapsulation of living cells and therapeutic proteins. At body temperature, however, they are able to form monolithic gels. In vivo tests showed that liquid formulations were converted into gel implants in situ. Thus, this system could be successfully used to deliver biologically active growth factors as well as an encapsulating matrix for living chondrocytes applicable in tissue engineering [71].

\subsection{Light-, electric- and magnetic- responsive hydrogels}

Electric, magnetic fields, and light exposure are the main external physical stimuli used to tune drug release kinetics from smart hydrogels. Once these exogenous stimuli can be controlled, they have the advantage of modulating drug release spatially and temporally [72-80].

Among the given classes of light-responsive systems, the stimulus source used can be derived from visible, near infrared (NIR) or ultraviolet (UV) light. There are two types of UV light responsivity: photopolymerization and photocleavage systems.

Photopolymerization consists of the gelification process of a liquid polymeric formulation to obtain a hydrogel system. Although it has been used as one stage in hydrogel synthesis, photopolymerization can also be used to induce in situ gelation [81-85]. In order to occur photopolymerization, photoinitiators, such as benzoin derivatives, benziketals acetophenone, and hydroxyalkylphenones, must interact with visible or UV light to form free radicals $[86,87]$. Another approach is the functionalization of hydrophilic polymers with photosensitive molecules, such as cinnamate, coumarin, or benzyl diethyldithiocarbamate, which are able to undergo intermolecular photodimerization after UV light exposure [88-90].

Nitrocinnamate-derived polyethylene glycol (PEG-NC) in situ hydrogels were developed for fibroblast growth factor (bFGF) incorporation. UV light ( $365 \mathrm{~nm}$ ) was used to promote photo-crosslinking of PEGNC. The cross-link density and the degree of swelling were influenced by the UV exposure time, modulating the drug release profile [82].

On the other hand, the main principle of the photocleavage for light-responsive hydrogels is based on the use of cross-linkers that exhibit photosensitivity (e.g., o-nitrobenzyl derivatives and azobenzene). These molecules, after exposure to light, undergo either photoisomerization or photooxidation, which lead to changes in the hydrogel matrix structure and, consequently, in drug or biomolecule release [74,91-94]. Irradiation with UV light at long wavelengths (365 nm) or visible light $(400-500 \mathrm{~nm}$ ) leads to irreversible photoisomerization of the $o$-nitrobenzyl ether group. However, some differences in the absorbance and quantum yield of this photosensitive crosslinking agent at different wavelengths, result in different degradation rates. Therefore, the choice of wavelength can be very important to control the release of drugs from this responsive system [74].

It is important to note that UV light holds some disadvantages when used as a physical stimulus for smart hydrogel drug delivery. UV has poor penetration into some body parts, and it can be potentially carcinogenic, especially under long-term exposure [80,95,96]. One of the strategies used to overcome these problems is adopting smart hydrogels containing up-converting nanoparticles (UCNPs) as the UV light source. This is an alternative because UCNPs are able to convert photon energy, absorbed from NIR light, into UV light [97,98]. Thus, the cross-linked photosensitive molecules absorb the UV light to initiate the gel-sol process, triggering the release of drugs [72,91,95]. An important parameter to be considered about these systems is the distribution of the photocleavable cross-linker molecules and the UCNPs. Yan and coworkers (2012) have incorporated UCNPs into a polymeric bulk hydrogel, while Jalani and co-workers (2015) coated UCNP particles with hydrogel. Based on the structural arrangements and reflected in laser power densities and laser time exposures, these disparities trigger 
different drug release profiles from the hydrogel matrix [91,95].

Electric fields are also used as external physical stimuli to modulate drug release from smart hydrogels. These systems, termed electroconductive hydrogels (ECHs), are developed from either polyelectrolytes $[99,100]$ or from an intrinsically conducting polymer (ICP) within a tridimensional cross-linked polymer network [79,101]. When an electric field is applied, driven charges are moved. Thus, ICPs can undergo electrochemical oxidation/reduction, leading to structural changes in the hydrogel and, subsequently, polymeric network erosion to release the incorporated drug $[79,101,102]$. Drug release from electro-responsive hydrogels can be related to drug charge, as shown by Choi and co-workers (2015). In their study, tetracycline was incorporated into carbon nanotube (CNT)-incorporated polyvinyl alcohol (PVA)-based hydrogels. At $\mathrm{pH}$ 8.0, tetracycline becomes negatively charged, and therefore, faster release was observed when a negative electric current was applied. This effect is due to repulsion between the drug and the negative bias. However, when a positive electric current was used, release was slower [101].

Several published studies have reported high-frequency magnetic fields (HFMFs) as external stimuli for drug delivery; a strategy which aims to accumulate these systems in an affected area. HFMFs are less invasive than electric fields or light exposure methods because they are able to highly permeate tissues with low energy absorption.

Magnetically responsive hydrogels are developed by incorporating magnetic iron oxide nanoparticles (MIONs), also known as super magnetic iron oxide nanoparticles (SPIONs), into the polymer matrices. They are widely explored, especially in association with thermo-responsive hydrogels.

The use of MIONs, incorporated to responsive polymers, is based on their capacity to transform electromagnetic energy, from an external high-frequency field, into heat. This event is related to the hysteresis that occurs, which can activate specific responses, such as drug release in a controlled manner [103,104]. As an example, ferrogel systems containing MIONs were developed by Hu and co-workers (2007), under HFMFs. They were activated kinetically and thermally (for large nanoparticles) promoting microstructural and molecular modifications, such as shrinking of the gelatin matrix, a thermosensitive polymer, which can control the drug release from the network with desirable precision [105]. An important parameter to be considered in these systems is the polymer/MIONs ratio. Sometimes, increased polymer/ MIONs ratio reduces the saturation magnetization value, and consequently, the drug release decreases under a magnetic field [106].

\section{3. pH-responsive hydrogels}

The human body exhibits substantial $\mathrm{pH}$ changes in different body parts when functioning normally (Fig. 4). Within women's bodies, while healthy vaginal mucosa is an acidic environment ( $\mathrm{pH} 4.0-5.0)$ the colon exhibits a $\mathrm{pH}$ value ranging from 7.0 to 7.5. In addition to the significantly different $\mathrm{pH}$ values exhibited in different tissues, some disease states can also cause $\mathrm{pH}$ changes in the human body [41]. The $\mathrm{pH}$ spectrum among different sites represent attractive targets for biomedical and pharmaceutical fields by which pH-responsive hydrogels could respond to a dynamic environment [42]. Moreover, $\mathrm{pH}$ changes might be easily manipulated and are applicable for in vitro and in vivo conditions [107].

Chemical stimuli, such as $\mathrm{pH}$ change, directly affect the interactions between polymer chains and between polymer chains and solvent molecules [108]. Similar to macromolecules, such as protein and nucleic acids, a polymer network contains ionizable groups that can donate or accept protons in response to $\mathrm{pH}$, which primarily results in solubility and structural changes and, consequently, swelling or deswelling. Brønsted acids and bases are mostly used as respective pendants since their protonation/deprotonation occur at $\mathrm{pH}$ values between 4.0 and 8.0. Charged polymers in aqueous solution are polyelectrolytes, which might adopt expanded conformation as a result of electrostatic repulsion, enabling the hydration process. The transition point is governed by the $\mathrm{p} K_{\mathrm{a}}$ value, and when the $\mathrm{pH}$ of the medium approaches the $\mathrm{p} K_{\mathrm{a}}$, the ionization degree of the polymer suffers a dramatic change [108]. Furthermore, charge exposition in aqueous medium is guided by the presence of carboxylic and amine groups that designate anionic and cationic behavior, respectively, two different classes of $\mathrm{pH}$ responsive hydrogels $[22,42]$.

The cross-linking process between responsive polymeric chains results in the self-assembly of smart hydrogels, changing its hydrodynamic volume according to the $\mathrm{pH}$ value. The swelling of polyelectrolyte hydrogels occurs most often as a result of electrostatic repulsion between charges on the polymer backbone, which is directly related to $\mathrm{pH}$ alterations [21]. Mechanical properties and turbidity can also be changed by $\mathrm{pH}$ modifications [108]. Despite the system alterations promoted by $\mathrm{pH}$, it is important to highlight that a dynamic response is essential for their applicability [21]. The critical $\mathrm{pH}$ value that determines their responsiveness can be modified by the incorporation of hydrophobic moieties, generally by copolymerization with hydrophobic monomers [108]. Amino acid residues can also be introduced on synthetic polymer chains to induce responsiveness through carboxyl groups.

pH-responsive polyacids almost always carry carboxylic groups and exhibit $\mathrm{p} K_{\mathrm{a}}$ values in the range of 5.0-6.0. At higher $\mathrm{pH}$ values, the carboxylic group is deprotonated, yielding polyelectrolytes and electrostatic repulsion between molecular chains. On the other hand, at low $\mathrm{pH}$, they are maintained as non-ionized macromolecules [108]. The most common examples are described in Table 4. Fig. 5 illustrates a hydrogel consisting of acid polymer chains that are responsive to $\mathrm{pH}$ variations in the surrounding medium.

Studies have frequently reported that synthetic polyacid monomers include acrylic acid, methacrylic acid, maleic anhydride, N,N-dimethylaminoethyl methacrylate and sulfonamide-containing polymers [109-112]. The pH-sensitive polyacids containing sulfonamide groups exhibit $\mathrm{pK}_{\mathrm{a}}$ values ranging from 3.0 to 11.0 , which enables the ionization of the hydrogen atom from the amide nitrogen [112].

Amine groups from polybases release protons under basic conditions, but accept them at low $\mathrm{pH}$ values yielding polyelectrolytes (Table 4). Examples of weak polybases are poly(N,N-dimethyl aminoethyl methacrylate), poly(N,N-diethyl aminoethyl methacrylate), aromatics 4-vinylpyridine, 2-vinylpyridine, poly (vinyl imidazole), and poly(L-lysine) [108,112-114].

pH-responsive behavior can also be found among natural polymers such as alginate, albumin, chitosan, pectin and gelatin [115-118]. When comparing synthetic homologues to natural patterns, it is necessary to highlight the high capacity of natural polymers to degrade in the body, an ideal feature for drug delivery platforms [119].

Chitosan and alginate are representative natural polybase and polyacid polysaccharides, respectively, that suffer physical crosslinking through hydrophobic or charge interactions. Swelling or liquid expulsion occurs based on the availability of the ionized group along the polymer chain. Ionized groups at high densities promote charge accumulation and, subsequently, electrostatic repulsion, and swelling. For chitosan, protonation of amino groups under low $\mathrm{pH}$ promotes electrostatic repulsion between cationic segments [120,121]. Pectin and alginate are anionic polysaccharides found in polyacid biodegradable hydrogels; alginate being composed of both guluronic acid (G) and mannuronic acid (M); whereas pectin contains galacturonic acid, which provides abundant carboxylate and hydroxyl groups. This feature yields high hydrophilicity and polymer chain extension by charge repulsion according to the $\mathrm{pH}$ [119]. While homopolymers based on weak polyelectrolytes appear to be $\mathrm{pH}$ responsive, the majority of these smart systems are developed by the combination of functional domains to achieve ideal responsiveness. Thus, the association between biopolymer and synthetic chains should be taken into account [112].

Due to its rigid crystalline structure, chitosan exhibits low solubility in water and in biologic fluids $(\mathrm{pH} 7.4)$, which might limit its use in 


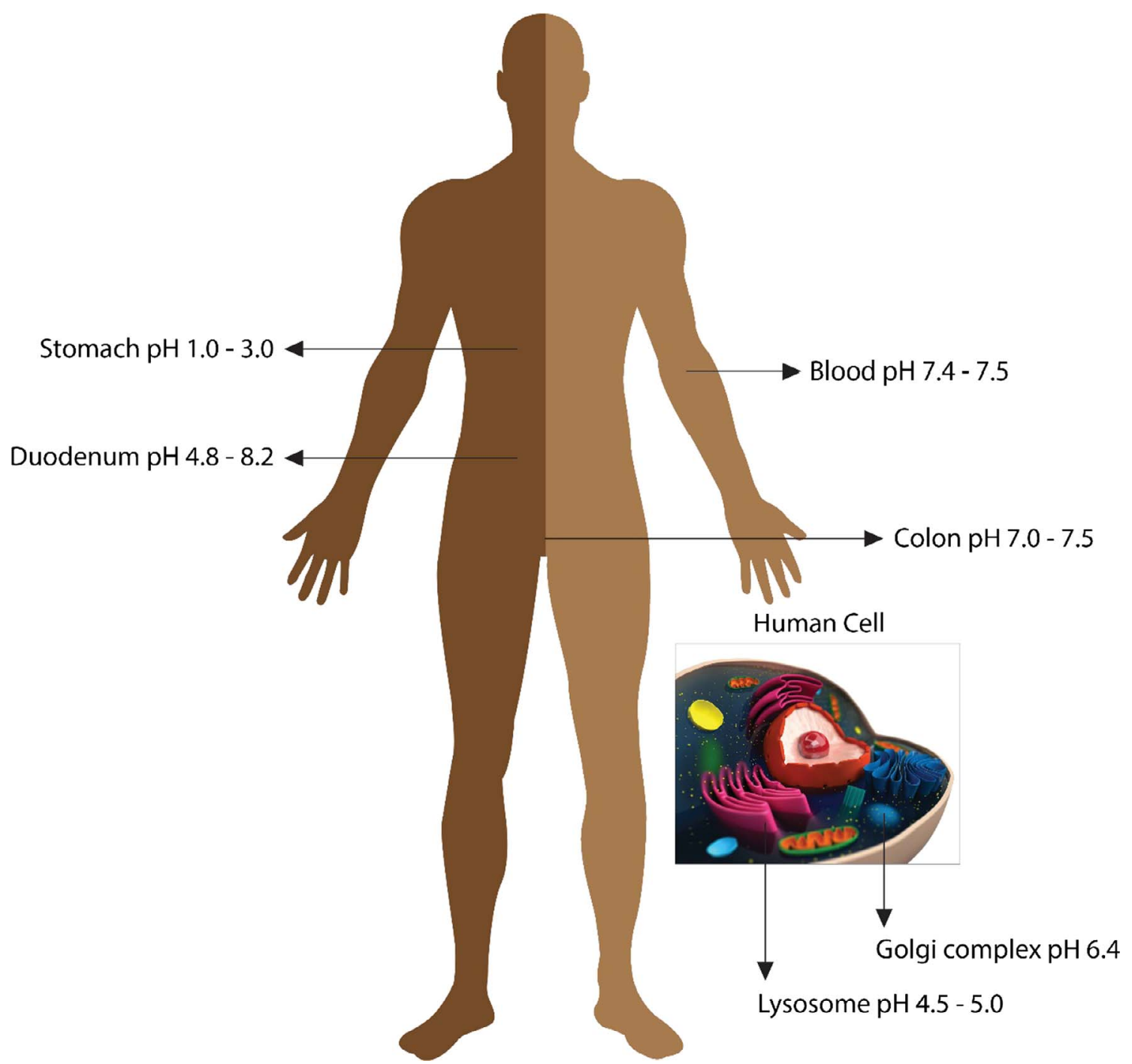

Fig. 4. $\mathrm{pH}$ values in several healthy tissues and cell compartments.

hydrogel development. However, a variety of chitosan copolymers can overcome this limitation, allowing the design of $\mathrm{pH}$-responsive systems [122]. Given its excellent biocompatibility and biological properties, carboxymethyl chitosan (CM-CS) demonstrated good solubility in a wide range of $\mathrm{pH}$. This was due to the additional presence of $\mathrm{COOH}$ groups, provided by substitution, which also governs their swelling behavior. Thus, the CM-CS have great potential as an oral drug delivery system that allows drugs to reach the colon [107]. CM-CS crosslinking with glutaraldehyde was used to design a pH-responsive delivery system, which enables higher $\mathrm{pH}$-dependent swelling and drug release in a controlled manner at intestinal $\mathrm{pH}$ [115].

One of the most exploited applications of pH-responsive systems is the development of oral platforms since oral delivery requires drug resistance to enzymatic attacks and to the $\mathrm{pH}$ gradient along the gastrointestinal tract [107]. The use of copolymers consisting of methacrylic acid (MAA) and grafted poly(ethylene glycol) (PEG) chains, known as P(MAA-g-EG), was widely explored by Peppas and co-workers to develop an oral delivery platform for therapeutic proteins. The developed network showed $\mathrm{pH}$-responsive behavior linked to the MAA polymer, which exhibited a $\mathrm{p} K_{\mathrm{a}}$ of 4.8. Watkins and Chen synthesized lysine-based hydrogels from poly(L-lysine isophthalamide) (PLP) crosslinked with L-lysine methyl ester to create a pH-responsive platform for oral administration of pharmaceutical drugs (ranging from 0.3 to $2000 \mathrm{kDa}$ ) to target the colon. In acidic environments, PLP carboxylic groups are protonated, promoting network collapse and drug retention. In the small intestine, these groups become negatively charged, and the three-dimensional structure absorbs a large amount of water, allowing for drug release [123]. Biodegradable and $\mathrm{pH}$-sensitive hydrogels composed of polyacrylic acid derivatives (PAAD) and poly(L-glutamic acid) (PGA), synthesized by Gao and co-workers, showed interesting properties for their use as insulin drug delivery by oral administration [124].

\subsection{Salt- or ionic strength-responsive hydrogels}

Based on different salt concentrations, some polymers undergo structural changes, which make them ionic strength- or salt-responsive systems [125]. Some studies have demonstrated that some polymer conformational transitions can be induced by different salt species and concentrations in the surrounding medium [126]. Similar to the previously described classes of $\mathrm{pH}$-responsive polymers, this proposed mechanism of responsiveness is based on increased hydrophobic interactions promoted by salt concentrations which results in a reduction of electrostatic repulsion between the copolymers, enabling network precipitation [112]. Although sodium phosphate or sodium sulfate buffers promote a gradual reduction of hydrogel swelling, this behavior is not associated with phase transition [125].

This feature can be extremely important because some biological 
Table 4

Molecular structure of common pH-responsive polyacids and polybases.

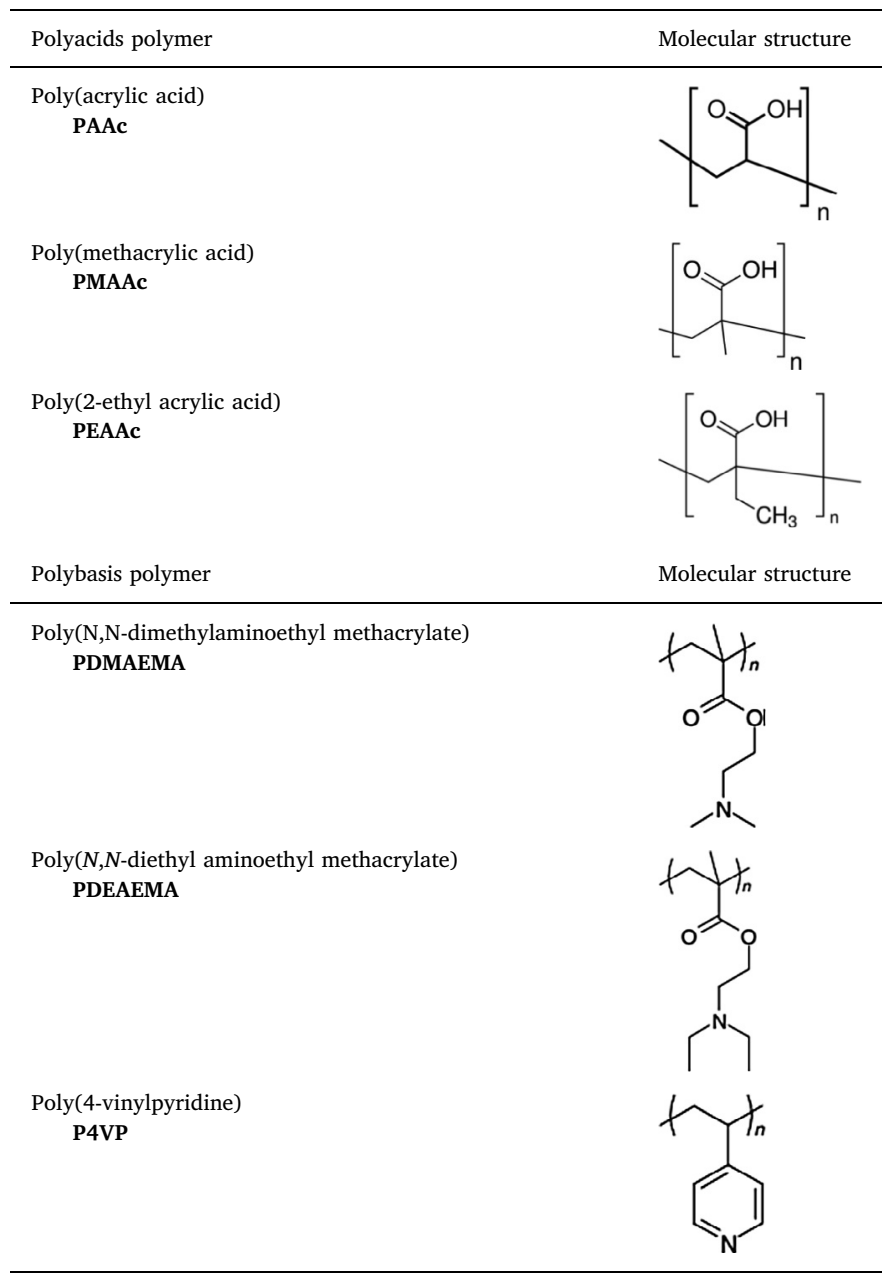

processes, such as nerve excitation, muscle contraction, and cell locomotion, involve ionic strength modifications [127]. However, these polymers are less abundant than $\mathrm{pH}$ - and temperature-responsive polymers and, consequently, have not been widely explored as smart drug delivery systems $[7,128]$.

Some weak polyacids such as poly(acrylic acid) and methacrylic acid show an increased degree of ionization upon the addition of lithium methoxide salt, which promotes viscosity changes. The shrinkage of coils is explained by the attraction between the mobile osmotically ion pairs. Neutralized polyacrylate hydrogels exhibit reversible volume changes by the addition of both monovalent and divalent cations in a concentration similar to physiological conditions [127].

Hydrogels composed of N-isopropylacrylamide (NIPAAm), a nonionic polymer, exhibit a known lower critical solution temperature. Park and co-workers demonstrated that the NIPAAm polymer also showed a sharp volume phase transition depending on the sodium chloride concentration in the surrounding medium [126].

Polyaspartic acid has been used in hydrogels that have the capacity of high swelling. However, ionic hydrogels commonly have reduced swelling capacities in saline solutions, especially because of electrostatic repulsion and decreased osmotic pressure. Zhao and co-workers studied the swelling profile of these hydrogels in various chloride and sulfate salt concentrations. The results demonstrated that swelling capacity is related to the cation charge and that it sharply drops in the presence of multivalent cations. They also reported that this reduction can be related to the greater number of univalent cation atoms [129].

A poly(N-isopropylacrylamide) (PNIPAM) hydrogel with specific sensitivity was synthetized by Liu and co-workers by introducing dibenzo-18-crown- 6 comonomers into the network. The developed system exhibited thermal responsivity and a swelling ratio sensitive to the presence of $\mathrm{K}^{+}[130]$.

\subsection{Bioresponsive hydrogels}

Bioresponsive hydrogels, also called biologically stimulated or biomolecule responsive hydrogels, are biomaterials able to undergo structural modifications (swelling or deswelling, degradation or erosion and mechanical deformation) mediated by biological reactions. Changes might occur in response to increased concentrations of specific biomolecule availability (proteins, peptides, enzymes, antibodies) in physiological environments or under pathological conditions [131].

Living organisms utilize an arsenal of molecular mechanisms to maintain homeostasis, including tissue development and repair, immune responses and blood coagulation processes [132].

Antibodies and $\mathrm{T}$ cells are important biomolecules responsible for maintaining normal body functions by controlling immune responses and autoimmunity, extremely affected in diseases such as cancer. Enzymes, which are highly selective in their reactivity, are vital catalysts and highly efficient in a series of biological pathways [133] and can be used as important signals for disease diagnosis [134]. All of these
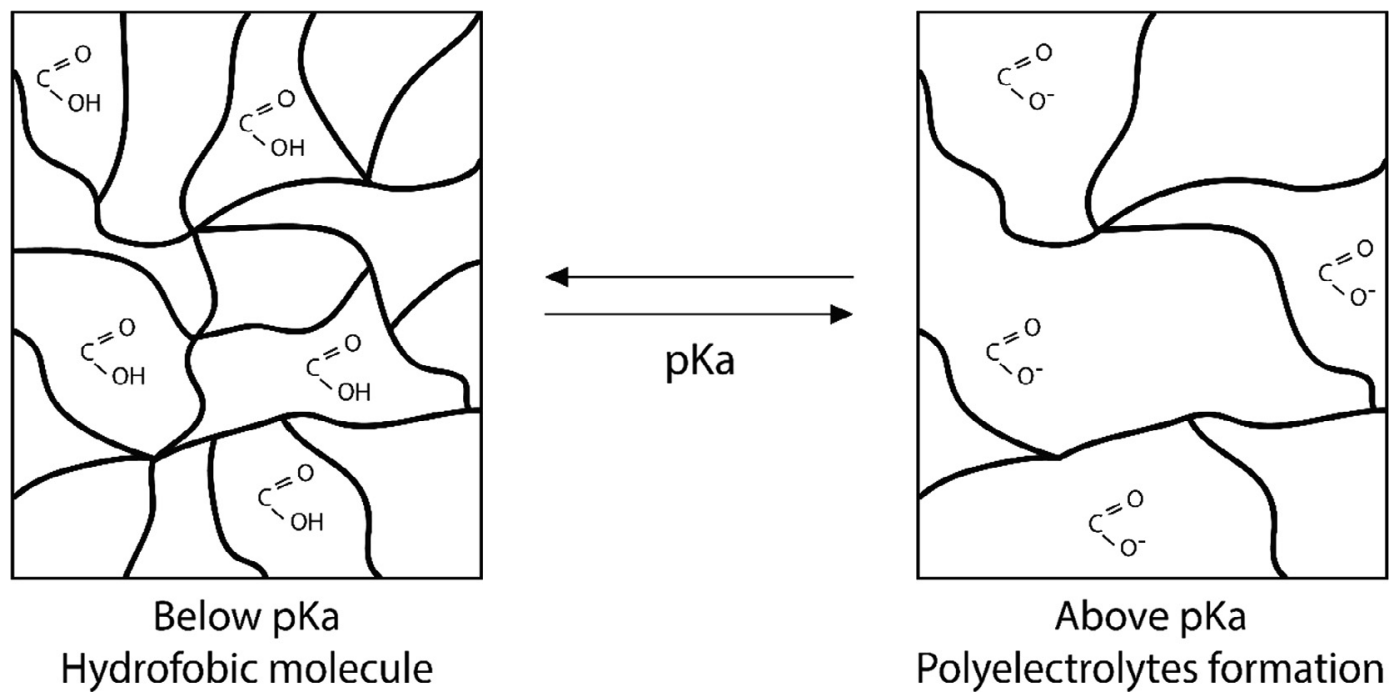

Fig. 5. A polyacid hydrogel response to surrounding medium $\mathrm{pH}$ variations. 
biomolecules may provide important signals to monitor biological functions or detect physiological changes.

The use of bioresponsive hydrogels can provide material platforms that recognize target biomolecules, such as biomarkers, promoting drug release or signaling transduction $[15,135,136]$. Many research groups have recently focused in the development of bioresponsive hydrogels as smart self-regulated biomaterial systems, useful not only for drug delivery [133,137-142] but also for diagnosis, cell cultures [15,135,136], biosensors, or matrices for tissue engineering and regenerative medicine [135].

Bioresponsive hydrogels can be classified according to their behavior and most of them exhibit three types of responsiveness. The first, is related to glucose-responsive hydrogels designed with entrapped insulin, which selectively bind glucose molecules, triggering a response according to blood glucose levels [37,143-145]. The second type, enzyme-responsive hydrogels, contain enzyme-sensitive substrates, such as short peptides, which can be programmed to respond to a specific enzyme [146]. This concept is especially attractive since enzyme distribution occurs differently in different body parts and, primarily, in healthy and in diseased environments. The third class is represented by antigen-responsive hydrogels, which exhibit antigens incorporated into their structural networks to recognize specific antibodies [133,147-150].

Glucose-responsive hydrogels are widely used in diabetes treatments [42], compensating for the inability of the pancreas to control glucose levels in the blood [135]. Furthermore, these systems can exhibit different strategies for insulin self-regulation: those that use glucose oxidase, lectin (concanavalin A) and phenylboronic acid $[131,132,151]$.

Glucose-responsive hydrogels designed with the glucose oxidase enzyme (GOX), entrapped along the polymer chain, show a mechanism of responsiveness to $\mathrm{pH}$ changes in the environment. This occurs due the cleavage of glucose molecules, followed by swelling/shrinking according to glucose levels. Essentially, when glucose concentration increases in the blood, GOX converts glucose to gluconic acid and $\mathrm{H}_{2} \mathrm{O}_{2}$, which lowers the $\mathrm{pH}$ inside the hydrogel. This reduction induces the ionization of functional groups and polymer chain repulsion inside the network, leading to hydrogel swelling and insulin permeation throughout the network reaching the blood $[42,47,135,136,152]$.

Hydrogels designed with entrapped GOX have been reported since 1963, when Bernfeld and Wan successfully immobilized these enzymes into acrylamide hydrogels. Later, in 1985, Albin and co-workers immobilized GOX into hydrogels based on N,N-dimethylaminoethyl methacrylate, hydroxyethyl methacrylate, and tetraethylene glycol dimethacrylate, obtaining a glucose-sensitive insulin delivery device. Since then, many studies have been performed with hydrogels using the same GOX pathway $[42,143,153]$. Recently, Traitel and co-workers showed that a glucose-responsive hydrogel based on the $\mathrm{pH}$-responsive poly(2-hydroxyethyl methacrylate-co-N,N-dimethylaminoethyl methacrylate) polymer, also known as poly(HEMA-co-DMAEMA), reached high swelling and insulin release rates when exposed to high levels of glucose concentration in the medium [145].

The second main pathway for glucose responsiveness consists of incorporating lectins, such as concanavalin A (Con A), into hydrogels [42]. Lectins are carbohydrate-binding proteins able to form complexes with glycoproteins and glycolipids present on the cell surface. Glucoseresponsive systems designed with entrapped Con A are able to control the release of glycosylated insulin by lectin-binding in response to free glucose $[135,152]$. These systems exploit the competitive binding behavior of Con A with glucose and glycosylated insulin, since Con A has a higher affinity for glucose than the glycosylated moieties. Therefore, expansion of the polymer chains leads to swelling and, consequently, insulin release by diffusion [152].

A hydrogel system composed of glucosyl-terminal PEG and insulin conjugates was covalently bound to Con A and then attached to a poly (vinylpyrrolidine-co-acrylic acid) backbone. Published work demonstrated that this system promoted controlled insulin release without greatly modifying the hydrodynamic hydrogel volume. Furthermore, the surface-modified hydrogel was able to load a large amount of drugs into the network [154].

The last main mechanism of glucose-responsive hydrogels is based on phenylboronic acid (PBA). The ability of PBA to sense glucose and to release insulin in a controlled manner is due to their affinity for polyol molecules (sugar alcohols). After recognizing glucose molecules, PBA is reversibly complexed with polyol compounds. The interaction between immobilized PBA and glucose leads to volumetric changes, which allows insulin release $[37,42,135]$. In aqueous environments, phenylborate groups exhibit an equilibrium between uncharged and charged forms. The reaction between PBA and glucose occurs through the cationic charged forms and, therefore, the reaction shifts the overall gel charge density toward cationic charged species as a consequence of the dissociation equilibrium. The increased charge into the hydrogel promotes polymers chain repulsion and increased hydrophilicity, leading to swelling [42,135]. In 1959, Lorand and Edwards were the pioneers to describe, in a quantitative study, the complexation of boronic acids and polyols. Since then, a series of studies has been performed to investigate the binding affinity of boronic acids with different diols, including fructose and glucose [144,155]. A novel glucose-responsive hydrogel system based on covalent dynamic bonds and inclusion complexation was developed using the poly(ethylene oxide)-b-poly vinyl alcohol (PEO- $b$-PVA) diblock polymer, $\alpha$-cyclodextrin ( $\alpha$-CD) and phenylboronic acid (PBA)-terminated PEO crosslinker. While dynamic covalent bonds between PVA and PBA provide sugar-responsive crosslinks, the inclusion complexation between PEO and $\alpha-\mathrm{CD}$, can provide hydrogel formation and hydrogel stability. PBA is an organoboronic acid capable of forming a dynamic complex with a polyol, such as PVA, however, in the presence of glucose, these complexes are dissociated once the biding constant of glucose-PVA is higher than PBA-PVA. Thus, the developed system provided drug release in response to glucose levels under physiological $\mathrm{pH}$ [39].

In cellular environments, most stimuli-responsive mechanisms are under the control of enzymes [156,157]. Thus, enzyme-responsive hydrogels can be a promising approach to respond directly to the target molecule. These systems can be designed based on peptidic building blocks; i.e., short sequences of amino acids, specifically degraded or digested by certain enzymes; which results in hydrogel degradation [42]. Since enzymes are highly selective in their reactivity, responsiveness is specific [135]. The enzyme access to self-assembled gels promote supramolecular architecture modifications based on the degradation process, which might result in swelling/shrinking or transformation of surface properties $[135,146]$. The first reported application related to tissue engineering was a metalloproteinase (MMP)sensitive PEG-based hydrogel developed for cartilage repair and chondrocyte culture. MMP-sensitive peptides were used as hydrogel crosslinkers, yielding a material that was specifically degraded by gelatinase and collagenase. Thus, this bioresponsive hydrogel exhibited a release rate that could be modulated by cellular enzyme secretion [158].

Enzyme-responsive hydrogels are also widely exploited to release drugs specifically in the colon. The use of $\mathrm{pH}$-sensitive monomers (acrylamide derivatives and acrylic acid) cross-linked with azo-aromatic bonds allows the degradability of the system to be restricted to the colon environment. Its responsiveness, in this case, is based on its ability of shrinking in the upper portions of the gastrointestinal tract, but when the system reaches the small intestine, where the $\mathrm{pH}$ increases, the swelling process begins. When the system arrives in the colon, the hydrogels reach their highest swelling degree. The crosslinked bonds are degraded by the action of azo-reductase enzymes or mediators. Subsequently, the hydrogel network can be degraded by cross-link cleavage, and the drug can be released at the desired site [159].

Hydrogels based on dextran polymers can also compose enzymeresponsive systems, since dextranases are present in colonic microbiota. 
These systems cross-linked with diisocyanate degraded both in a human colonic fermentation in vitro model and in an animal in vivo model. In addition, data showed that drug release from the hydrogel was dependent on the presence of dextranases in the release medium [160].

Biodegradable polymers include naturally derived polymers such as pectin, amylose, gellan gum, chitosan, chondroitin sulfate, alginate, and dextran; [161] and artificial non-biodegradable polymers such as PEG/ poly(ethylene oxide) (PEO), poly(2-hydroxyethyl methacrylate) and poly( $\mathrm{N}$-isopropylacrylamide), which are enzyme-cleavable networks that are promising materials for developing site-specific drug release systems by enzymatic decomposition $[134,136,161]$.

Antibodies are biomolecules able to recognize specific antigens and to establish contact through supramolecular interactions [133]. Thus, antigen-responsive hydrogels are able to undergo volume or structural changes in the presence of antibodies [133,135]. Miyata and coworkers produced the first antigen-responsive hydrogel by coupling rabbit immunoglobulin G (rabbit IgG) to N-succinimidyl acrylate (NSA) [147]. When the antigen-responsive hydrogel was placed in a buffer solution containing antibodies cross-link density decreased due to the dissociation of the antigen-antibody binding in the presence of free antigen. Therefore, the authors observed that system swelling was dependent on the antigen concentration [145]. Lu and co-workers developed an antigen-responsive hydrogel by using a polymerizable antibody Fab' fragment, obtained from the monoclonal anti-fluorescein BDC1 antibody (IgG2a). This antibody was copolymerized with $\mathrm{N}$-isopropylacrylamide (NIPAAm) and $\mathrm{N}, \mathrm{N}^{\prime}$-methylenebis (acrylamide) (MBAAm; cross-linker) using redox initiators. The hydrogel showed reversible volume changes, and the antigen responsiveness was dependent on the Fab' content, $\mathrm{pH}$, and temperature [148]. This field of research continues to grow as new compositions of responsive polymer are developed in the next generation of smart materials.

\section{Smart hydrogels as functional delivery systems}

Smart hydrogels by nature can represent a model system for studying dynamic interactions between cells, between cells and extracellular matrixes, and between drug delivery and biological systems, as all these interactions can be of dynamic nature. However, the application of these bioactuating systems requires a sophisticated molecular design, which increases their potential applications [162]. Once it is a complex process in its entirety, only a deep knowledge of target pathology, science of the polymeric materials and therapeutic strategies provide the systemic reasoning required to design these systems.

Regarding material science and biological systems, new science is concerned with the design of smart materials for biological applications. To evolve in this field, we should gather multidisciplinary knowledge for this specific purpose [163,164].

Some diseases are known for significant changes on the homeostasis state. The evaluation of changes that characterize a pathological condition enables the exploitation of timely responsiveness to a particular biological event, as intrinsic properties constitute the basis for designing smart delivery systems [164]. In this sense, a smart system can be initially designed from target pathology characteristics. Here, we highlight some pathological aspects that can be explored toward this objective.

\subsection{Smart systems designed for inflammatory processes}

An inflammatory process is an organism's response to tissue injury or infection and involves the coordinated action of immune system cells and the release or activation of mediators such as growth factors, cytokines, chemokines, eicosanoids, biogenic amines and neuropeptides. A series of successive events promotes increased vasodilation and capillary permeability at the inflammation site, resulting typical inflammatory signs, such as pain, heat, redness and swelling $[165,166]$.

This process is divided into two response patterns: acute and chronic. Acute events are typically of short duration (minutes, hours or days) and are triggered by the activation of local and migratory cells, such as mast cells, macrophages, neutrophils, and monocytes with, local stimuli that lead to the expression of interleukins and tumor necrosis factors. On the other hand, chronic responses have last longer and involve marked cell migration, such as lymphocytes and macrophages, and the proliferation of vessels, fibrosis and tissue necrosis $[167,168]$.

Maintenance of the inflammatory process for an extended period can result in the development of several chronic diseases [168]. Rheumatoid arthritis, for example, is an autoimmune disease with a worldwide prevalence of $1 \%$, particularly affecting people from 30 to 55 years old. Its pathology is characterized by an intense inflammatory response in the synovial cavity of joints, which occurs due to the recruitment of inflammatory cells, such as T and B lymphocytes, dendritic cells, macrophages, neutrophils and fibroblasts [169]. Activated T cells proliferate and stimulate the production of inflammatory cytokines, which work as a stimulus for macrophage and fibroblast activation. In addition, secrete tumor necrosis factor (TNF- $\alpha$ ) and interleukins (IL-1, IL-6, IL-15 and IL-18) are mediators that trigger the migration of neutrophils to the inflammatory area.

As a consequence, inflammatory processes trigger hypertrophy of the synovial membrane, angiogenesis, and drastically increase synovial fluid volume, intracapsular pressure and temperature. Another feature concern the alterations of the synovial liquid: in physiological conditions, the synovial liquid is clear, light yellow and viscous with a $\mathrm{pH}$ ranging from 7 to 7.8. In pathologic states, the synovial liquid becomes turbid and acidic [170]. The enzyme production and lower $\mathrm{pH}$ contribute to accelerated tissue degradation, as well as cartilage and bone erosion [171].

Taking into account the aforementioned inflammatory particularities, the development of responsive drug delivery systems as hydrogels for intra-articular administration represents an advantageous strategy for treating chronic inflammation directly at the affected joint. These systems enable high drug concentrations at the desired site with limited systemic toxicity. Especially for rheumatoid arthritis, smart features might explore the following aspects: $\mathrm{pH}$ changes (in synovial liquids) and temperature (explored through the differences between ambient and body and also between regions of healthy and inflamed tissues). Furthermore, the presence of many enzymes at the inflammation site can be taken into account.

Thermo-responsive hydrogels might represent an interesting alternative because they are liquid at room temperature, which facilitates administration by site injections, and form a compact mesh when in contact with intra-articular temperatures equal to or higher than $37^{\circ} \mathrm{C}$ due to the inflammatory process. This ability allows local retention for extended periods of time in addition to controlled drug release [172]. Other important approaches include using the presence of many enzymes at the inflammation site to control drug release and hydrogel degradation [173]. Some catalytic enzymes present at the inflammation site are uniquely chemo-, region-, and enantioselective and function under inflammatory conditions $\left(\mathrm{pH} 5-8,>37^{\circ} \mathrm{C}\right)$ [174]. Hyaluronic acid, dextran, poly(ethylene glycol), PEO and PLA polymers are examples that can be used to develop this kind of responsiveness $[167,175]$.

Smart hydrogels may be a promising strategy for treating inflammatory bowel disease (IBD), which consists of Crohn's disease and ulcerative colitis [176]. Mucus depletion, in situ accumulation of positively charged proteins, and the presence of esterases and matrix metalloproteinases are characteristics of IBD that have been exploited as targets in the development of smart systems [177-179]. As an example, a smart hydrogel was developed to provide a system able to interact with mucus oligosaccharides by electrostatic forces, and promote drug release through the hydrolysis process, induced by esterases. Therefore, the developed hydrogel was able to adhere to the inflamed mucosa and act as a reservoir to promote sustained drug release [179]. 
Zhou and co-workers developed supramolecular smart nanofibers/ hydrogels to repair the colonic mucosa and reduce the inflammatory process. The main strategies used were: (1) specific drug release from hydrogels after azoreductase activity occurred in the colonic microflora; (2) D-peptides to increase the resistance to proteases, so the hydrogel system could reach the colon intact following oral administration; and (3) dynamic interactions from self-assembly providing flexibility and system adaptation to a series of mucosal surfaces [180]. The association of these strategies highlights the importance of understanding the microenvironment of the disease to engineer effective delivery systems.

\subsection{Smart systems designed for cancer therapy}

Cancer is one of the most aggressive diseases responsible for death around the world. For decades, chemotherapy has been the main treatment used. However, most of the chemotherapeutic agents are non-specific and exert several toxic side effects, which limits the dose that can be used and jeopardizes the patients' quality of life [181]. Based on the absence of consistent disparities between pathological and normal tissue, designing effective therapies for malignant diseases, such as cancer, has faced great difficulties [182]. However, some differences in metabolic and nutritional events, among a series of solid tumors and the surrounding normal tissue, can be observed. The accelerated increase in the population of expanding tumor cells is not supported by normal vasculature, leading to oxygen and nutrient deficiencies. Thus, tumors exhibit increased proliferation and angiogenesis [183,184].

First described by Otto Warburg, selective and active processes occur to provide adjustments on energy metabolism during uncontrolled cell proliferation and cancer progression. One of these events consists of shifting from oxidative phosphorylation to aerobic glycolysis for ATP production, even in the presence of oxygen [185,186]. As a result of high glycolytic metabolism rates, high levels of lactate/ $\mathrm{H}^{+}$ (lactic acid) are produced. Furthermore, the preservation of enhanced glycolytic flux and intracellular physiological $\mathrm{pH}$ is performed by the efflux of lactic acid to the extracellular microenvironment, preventing intracellular acidosis, which might result in cell death [182,187]. This condition results in acidic $\mathrm{pH}$ at the tumor microenvironment. Although, sometimes this characteristic is seen as a disadvantage due to drug permeation difficulties and to facilitating tumor invasion, it can also represent a target for developing smart hydrogels [188]. Therefore, $\mathrm{pH}$-responsive systems that effectively deliver anticancer drugs might carry and stabilize the therapeutic agent at physiological $\mathrm{pH}$, in addition to releasing the drug at this condition [189]. Numerous hydrogel systems have been proposed in this sense, particularly for local administration, where antitumor drugs can be directly transported to the cancer microenvironment [122,190-192].

The first aspect to be taken into consideration before designing a $\mathrm{pH}$-responsive hydrogel is the target $\mathrm{pH}$. Thereafter, the $\mathrm{p} K_{\mathrm{a}}$ value of the polymer chain should determine its applicability. The utilization of weak polyacids, such as the alginate polymer, to design $\mathrm{pH}$ responsive systems may be interesting for this approach [193]. In acidic environments, according to the polymer $\mathrm{pKa}$, alginate chains represent a matrix surrounded by negative charges, which are able to interact with positively charged drugs through supramolecular interactions. The acid environment makes these polyelectrolyte complexes stronger and more stable, which results in different drug release profiles, according to the $\mathrm{pH}$ changes at the tumor environment $[190,194]$.

Such pH-responsive systems might also be beneficial in intracellular microenvironments. It is well known that cell internalization occurs primarily through the endocytic mechanism. This event results in system confinement inside an acidic endosomal and lysosomal lumen. Exploring this mechanism, nanogels exhibit interesting results as smart systems $[195,196]$.

An additional striking feature that should also be explored in cancer therapy is based on the fact that an association between inflammation and cancer has been proposed [197]. Therefore, all previously described events considered regarding inflammatory processes may also be useful for developing smart systems for cancer therapy. Changes in tumor vasculature permeability and cell membranes, lower hydrostatic pressure and increase of localized temperature are conditions to be taken into account.

Considering the spontaneous hyperthermia in tumoral tissues, a temperature-responsive system based on chitosan-poly( $\mathrm{N}$-isopropylacrylamide-co-acrylamide) polymer was developed by Wang and co-workers to explore tumor microenvironments. Their results demonstrated the release of the drug in a temperature-dependent manner that favored cellular uptake against the tumor hyperthermia condition [198]. Furthermore, differences between the internal and external body temperatures have been extensively explored for localized therapy [199-202].

Another factor to consider is that the presence of some enzymes, such as esterases, can be explored to project responsiveness [192]. MMPs are zinc-dependent endopeptidases and are responsible for cleaving a series of extra-cellular matrix components. It is known that, in normal tissues, MMPs are expressed in minute amounts, whereas in tumoral tissues, especially in advanced stages of cancer, they are overexpressed [203]. In particular, MPP2 is associated with tumor progression, angiogenesis and metastasis. Thus, MPPs can therefore be used to promote responsiveness alone or in combination with other strategies [204-206].

Among all the tumor characteristics that can be explored to develop smart hydrogels for cancer therapy, $\mathrm{pH}$ differences are most explored to target tumor cells [188]. However, systems specifically oriented by $\mathrm{pH}$ changes are still limited compared to receptor targeting or endosomal $\mathrm{pH}$ targeting. This reality is justifiable due to the narrow $\mathrm{pH}$ window between normal and pathologic tissue. To complement this approach, more data on the changes of system kinetics are needed, which would allow the necessary adjustments for this purpose.

\subsection{Smart systems designed for diabetes mellitus}

Diabetes mellitus is a worldwide public health problem, which consists of a group of metabolic diseases characterized by hyperglycemia resulting from insulin secretion defects, insulin action, or both. In 2012, approximately 1.5 million deaths were directly related to diabetes, of which $43 \%$ occurred to individuals below the age of 70 . In 2014, statistics showed that approximately 422 million people were diabetic, representing approximately $8.5 \%$ of the adult population. Recently, the World Health Organization (WHO) estimated that the worldwide number of deaths related to diabetes is approximately 2.9 million/year, and this number is expected to increase by approximately $50 \%$ in the next 10 years $[207,208]$.

Under physiological conditions, increased glucose levels in the blood trigger a cascade of events that result in the synthesis and secretion of insulin by $\beta$-cells. Thus, insulin promotes decreased glucose levels by increasing glycogenesis and glucose uptake. When the blood glucose level returns to normal, the amount of insulin in the blood decreases [208].

The WHO recognizes two main forms of diabetes mellitus: type 1 and type $2[208,209]$. Type 1 diabetes is most common among children and is characterized by the lack of insulin production as a consequence of autoimmune destruction $[208,210]$. Type 2 diabetes generally occurs in middle-aged people, and insulin resistance is its main defect, which is characterized by low responses of target tissues to insulin [208,211,212].

Traditionally, diabetic patients, especially type 1 patients, have been treated by regularly applying subcutaneous insulin injections. This procedure is quite inconvenient, painful, can trigger hyperinsulinemia and allergic reactions and it is known low patient compliance. In addition, conventional medicines administered through alternative routes, such as dermal, rectal, ocular, oral, nasal, and pulmonary 
methods, are usually accompanied by low bioavailability. Therefore, innovative drug delivery systems have been intensively researched. Today, it is already possible to predict the production of responsive systems that are able to release insulin in response to blood glucose levels. In this context, the design of new drug delivery systems, such as smart bio-hydrogels, represent a promising strategy [208].

Based on glucose sensing and recognition strategies, some hydrogels show properties that can facilitate insulin diffusion. The described materials undergo a variety of changes, such as swelling, shrinking or degradation, in response to glucose, and each approach may offer a possible benefit [213].

Diabetes pathology and its particularity allows for the exploration of adjoining features to produce smart systems. For instance, although we know insulin peptides are heavily damaged at the acidic stomach, orally administered smart hydrogel systems might mitigate this problem, providing the necessary drug protection in the acidic environment and allowing drug release in lowers parts of gastrointestinal tract. Biocompatible and biodegradable natural polysaccharides are interesting materials to be used for these purposes. Blends of different polysaccharides enable the modulation of physical-chemical properties for the desired drug release profiles. Therefore, the understanding of system dynamics along with pathological particularities might provide a realizable self-assembly system that is able to exert the desired therapeutic effects.

\section{Future perspectives}

Science is constantly in expansion and fundamentally, the introduction of new technologies creates challenges for those responsible for regulating aspects applied on these new products, particularly if they may cause risks to health and environment. The absence of a homogeneous and clear regulatory guidelines involving technologybased smart systems creates an unstable scenario where benefits can be confused with risks, hindering their clinical outcome.

We can reflect on how our standard of living has been improved by significant progress in many fields related to science and engineering. However, it is important to note that our lives have been changing very quickly, and scientific progress along with regulatory aspects must accompany these changes. In this context, the design of smart systems offers important advances and novel solutions to a series of diseases and life threatening health conditions. In this literature review, a series of smart hydrogels developed for various applications by scientists and technologists all over the world were discussed. However, while many scientific reports have been published, a low number of hydrogel products are currently being used in clinical trials. This time lapse reflects the need for optimized designs of advanced smart systems, that focus on important aspects of human diseases and therapy efficacy along with toxicity predictions and regulatory improvements.

Misleadingly, hydrogels are commonly prepared or synthetized first, and suitable applications are later investigated for that system. In current research practice, preparations have not yet been designed considering any particular application or biological condition. Therefore, this current approach promotes unavoidable gaps between properties and applications. Thus, stimuli-responsive systems should be projected at the molecular level before considering the desired biological application. However, it is very likely that synthetically produced materials will approach the complexity of biologically spontaneous events soon through the use of non-linear chemical reaction networks [214].

Foreseen to be highly promising, the nanoarchitectonics of smart hydrogels must be based on basic research and researchers should adapt the properties of the drug delivery systems to obtain the desired biological effects. Additionally, to accomplish material self-assembly and the stimulation of spontaneous processes, professionals must bear in mind all legal guidelines established by local authorities.

Researchers believe that the use of dynamic hydrogels as drug delivery systems is still in its initial stage. However, the importance of these systems remains incontestable and highly promising.

Currently, the association of stimulus responsiveness and other design strategies can provide synergistic effects for targeted drug delivery. Furthermore, the development of a successful smart hydrogel delivery system requires exquisite material engineering and should, now more than ever, also include a multidisciplinary approach to gather expertise from polymer scientists, medical doctors, chemists and pharmacists.

Further rational studies of function-based systems can lead to the development of innovative and promising drug delivery systems.

\section{Acknowledgements}

The authors would like to thank Fundação de Amparo e Pesquisa do Estado de São Paulo (FAPESP), Coordenação de Aperfeiçoamento de Pessoal de Nível Superior (CAPES), Conselho Nacional de Desenvolvimento Científico e Tecnológico (CNPq), Ferring Pharmaceuticals and INCT-NANOFARMA for financial support.

\section{Conflict of interest}

The authors declare no competing financial interests.

\section{Appendix A. Supplementary material}

Supplementary data associated with this article can be found, in the online version, at http://dx.doi.org/10.1016/j.eurpolymj.2017.12.004.

\section{References}

[1] J. Kopeček, Hydrogel biomaterials: A smart future? Biomaterials 28 (2007) 5185-5192.

[2] M.C.I.M. Amin, N. Ahmad, M. Pandey, M.M. Abeer, N. Mohamad, Recent advances in the role of supramolecular hydrogels in drug delivery, Expert Opin. Drug Deliv. 12 (2015) 1149-1161.

[3] S.J. Buwalda, K.W. Boere, P.J. Dijkstra, J. Feijen, T. Vermonden, W.E. Hennink, Hydrogels in a historical perspective: From simple networks to smart materials, J. Control. Release 190 (2014) 254-273.

[4] E.S. Dragan, Design and applications of interpenetrating polymer network hydrogels. A review, Chem. Eng. J. 243 (2014) 572-590.

[5] A.S. Hoffman, Hydrogels for biomedical applications, Adv. Drug Deliv. Rev. 54 (2002) 3-12.

[6] N.A. Peppas, P. Bures, W. Leobandung, H. Ichikawa, Hydrogels in pharmaceutical formulations, Eur. J. Pharm. Biopharm. 50 (2000) 27-46.

[7] Y. Samchenko, Z. Ulberg, O. Korotych, Multipurpose smart hydrogel systems, Adv Colloid Interface Sci. 168 (2011) 247-262.

[8] L.-W. Xia, R. Xie, X.-J. Ju, W. Wang, Q. Chen, L.-Y. Chu, Nano-structured smart hydrogels with rapid response and high elasticity, Nat. Commun. 4 (2013) 2226.

[9] A.K. Azad, N. Sermsintham, S. Chandrkrachang, W.F. Stevens, Chitosan membrane as a wound-healing dressing: Characterization and clinical application, J. Biomed. Mater. Res. Part B Appl. Biomater. 69 (2004) 216-222.

[10] J. Chen, H. Park, K. Park, Synthesis of superporous hydrogels: Hydrogels with fast swelling and superabsorbent properties, J. Biomed. Mater. Res. A. 44 (1999) 53-62.

[11] A.C. Jen, M.C. Wake, A.G. Mikos, Review: Hydrogels for cell immobilization, Biotechnol. Bioeng. 50 (1996) 357-364.

[12] A.K.A. Silva, C. Richard, M. Bessodes, D. Scherman, O.-W. Merten, Growth facto delivery approaches in hydrogels, Biomacromolecules 10 (2009) 9-18.

[13] S.C. Lee, I.K. Kwon, K. Park, Hydrogels for delivery of bioactive agents: A historical perspective, Adv. Drug Deliv. Rev. 65 (2013) 17-20.

[14] L.-Y. Chu, R. Xie, X.-J. Ju, W. Wang, Smart Hydrogel Functional Materials, Springer-Verlag, Berlin Heidelberg, 2013.

[15] S. Chaterji, I.K. Kwon, K. Park, Smart polymeric gels: Redefining the limits of biomedical devices, Prog. Polym. Sci. 32 (2007) 1083-1122.

[16] R. Masteikova, Z. Chalupova, Z. Sklubalova, Stimuli-sensitive hydrogels in controlled and sustained drug delivery, Med. 39 (2003) 19-24.

[17] W. Kuhn, Reversible Dehnung und Kontraktion bei Änderung der Ionisation eines Netzwerks polyvalenter Fadenmolekülionen, Experientia 5 (1949) 318-319.

[18] A. Richter, Hydrogel-based $\mu$ TAS, in: C.T. Leondes (Ed.), MEMS/NEMS Handbook Techniques and Applications, Springer, New York, 2006, pp. 141-171.

[19] W. Kuhn, B. Hargitay, A. Katchalsky, H. Eisenberg, Reversible dilation and contraction by changing the state of ionization of high-polymer acid networks, Nature 165 (1950) 514-516.

[20] A. Katchalsky, Rapid swelling and deswelling of reversible gels of polymeric acids by ionization, Cell Mol. Life Sci. 5 (1949) 319-320.

[21] S.K. Samal, M. Dash, P. Dubruel, S. Van Vlierberghe, Smart polymer hydrogels: 
Properties, synthesis and applications, in: M.R.A. De Armas, J.S. Román (Eds.), Smart Polymers and their Applications, Woodhead Publishing, Cambridge, 2014, pp. 237-270.

[22] H. Zheng, L. Xing, Y. Cao, S. Che, Coordination bonding based pH-responsive drug delivery systems, Coord. Chem. Rev. 257 (2013) 1933-1944.

[23] J.-M. Lehn, Toward complex matter: Supramolecular chemistry and self-organization, Proc. Natl. Acad. Sci. 99 (2002) 4763-4768.

[24] J.-M. Lehn, Supramolecular chemistry, Science 260 (1993) 1762-1763.

[25] J.-M. Lehn, Constitutional dynamic chemistry: Bridge from supramolecular chemistry to adaptive chemistry, in: M. Barboiu (Ed.), Const Dyn. Chem. Springer, Berlin Heidelberg, 2012, pp. 1-32.

[26] J.-M. Lehn, Dynamers: Dynamic molecular and supramolecular polymers, Prog. Polym. Sci. 30 (2005) 814-831.

[27] A.V. Davis, R.M. Yeh, K.N. Raymond, Supramolecular assembly dynamics, Proc Natl. Acad. Sci. 99 (2002) 4793-4796.

[28] T. Maeda, H. Otsuka, A. Takahara, Dynamic covalent polymers: Reorganizable polymers with dynamic covalent bonds, Prog. Polym. Sci. 34 (2009) 581-604.

[29] J.A. Burdick, W.L. Murphy, Moving from static to dynamic complexity in hydrogel design, Nat. Commun. 3 (2012) 1269.

[30] C.M. Kirschner, K.S. Anseth, Hydrogels in healthcare: From static to dynamic material microenvironments, Acta Mater. 61 (2013) 931-944.

[31] P. Sacco, M. Borgogna, A. Travan, E. Marsich, S. Paoletti, F. Asaro, M. Grassi, I. Donati, Polysaccharide-based networks from homogeneous chitosan-tripolyphosphate hydrogels: Synthesis and characterization, Biomacromolecules 15 (2014) 3396-3405.

[32] Z. Zhang, L. Chen, C. Zhao, Y. Bai, M. Deng, H. Shan, X. Zhuang, X. Chen, X. Jing, Thermo- and pH-responsive HPC-g-AA/AA hydrogels for controlled drug delivery applications, Polymer 52 (2011) 676-682.

[33] X. Qiu, Y. Yang, L. Wang, S. Lu, Z. Shao, X. Chen, Synergistic interactions during thermosensitive chitosan- $\beta$-glycerophosphate hydrogel formation, RSC Adv. 1 (2011) 282-289.

[34] S.T.K. Raja, T. Thiruselvi, A.B. Mandal, A. Gnanamani, pH and redox sensitive albumin hydrogel: A self-derived biomaterial, Sci. Rep. 5 (2015) 15977.

[35] S.-Y. Choh, D. Cross, C. Wang, Facile synthesis and characterization of disulfidecross-linked hyaluronic acid hydrogels for protein delivery and cell encapsulation, Biomacromolecules 12 (2011) 1126-1136.

[36] O.P. Oommen, S. Wang, M. Kisiel, M. Sloff, J. Hilborn, O.P. Varghese, Smart design of stable extracellular matrix mimetic hydrogel: Synthesis, characterization, and in vitro and in vivo evaluation for tissue engineering, Adv. Funct. Mater. 23 (2013) 1273-1280.

[37] C. Zhang, M.D. Losego, P.V. Braun, Hydrogel-based glucose sensors: Effects of phenylboronic acid chemical structure on response, Chem. Mater. 25 (2013) $3239-3250$.

[38] N. Boehnke, C. Cam, E. Bat, T. Segura, H.D. Maynard, Imine hydrogels with tunable degradability for tissue engineering, Biomacromolecules 16 (2015) 2101-2108.

[39] T. Yang, R. Ji, X.-X. Deng, F.-S. Du, Z.-C. Li, Glucose-responsive hydrogels based on dynamic covalent chemistry and inclusion complexation, Soft Matter 10 (2014) 2671-2678.

[40] F. Yu, X. Cao, J. Du, G. Wang, X. Chen, Multifunctional hydrogel with good structure integrity, self-healing, and tissue-adhesive property formed by combining Diels-Alder click reaction and acylhydrazone bond, ACS Appl. Mater Interfaces 7 (2015) 24023-24031.

[41] D. Schmaljohann, Thermo-and pH-responsive polymers in drug delivery, Adv. Drug Deliv. Rev. 58 (2006) 1655-1670.

[42] M.C. Koetting, J.T. Peters, S.D. Steichen, N.A. Peppas, Stimulus-responsive hy drogels: Theory, modern advances, and applications, Mater. Sci. Eng. R Reports 93 (2015) 1-49.

[43] H.P. Merkle, Drug delivery's quest for polymers: Where are the frontiers? Eur. J. Pharm. Biopharm. 97 (2015) 293-303.

[44] M.R. Aguilar, C. Elvira, A. Gallardo, B. Vázquez, J.S. Román, Smart polymers and their applications as biomaterials, in: N. Ashammakhi, R. Reis, E. Chiellini (Eds.), E-book Topics in Tissue Engineering, vol. 3, 2007 (chapter 6).

[45] L. Klouda, A.G. Mikos, Thermoresponsive hydrogels in biomedical applications, Eur. J. Pharm. Biopharm. 68 (2008) 34-45.

[46] Z. Lin, W. Gao, H. Hu, K. Ma, B. He, W. Dai, X. Wang, J. Wang, X. Zhang, Q. Zhang, Novel thermo-sensitive hydrogel system with paclitaxel nanocrystals: High drugloading, sustained drug release and extended local retention guaranteeing better efficacy and lower toxicity, J. Control. Release. 174 (2014) 161-170.

[47] Y. Qiu, K. Park, Environment-sensitive hydrogels for drug delivery, Adv. Drug Deliv. Rev. 53 (2001) 321-339.

[48] M.R. Matanović, J. Kristl, P.A. Grabnar, Thermoresponsive polymers: Insights into decisive hydrogel characteristics, mechanisms of gelation, and promising biomedical applications, Int. J. Pharm. 472 (2014) 262-275.

[49] E. Ruel-Gariepy, J.-C. Leroux, In situ-forming hydrogels-review of temperaturesensitive systems, Eur. J. Pharm. Biopharm. 58 (2004) 409-426.

[50] M. Prabaharan, J.F. Mano, Stimuli-responsive hydrogels based on polysaccharides incorporated with thermo-responsive polymers as novel biomaterials, Macromol. Biosci. 6 (2006) 991-1008.

[51] V.R. de la Rosa, R. Hoogenboom, Solution polymeric optical temperature sensors with long-term memory function powered by supramolecular chemistry, Chem. Eur. J. 21 (2015) 1302-1311.

[52] A. Gandhi, A. Paul, S.O. Sen, K.K. Sen, Studies on thermoresponsive polymers: Phase behaviour, drug delivery and biomedical applications, Asian J. Pharm. Sci. 10 (2015) 99-107.

[53] R. Pelton, Poly (N-isopropylacrylamide) (PNIPAM) is never hydrophobic, J.
Colloid Interface Sci. 348 (2010) 673-674.

[54] H.G. Schild, Poly (N-isopropylacrylamide): Experiment, theory and application, Prog. Polym. Sci. 17 (1992) 163-249.

[55] F. Meeussen, Y. Bauwens, R. Moerkerke, E. Nies, H. Berghmans, Molecular complex formation in the system poly(vinyl methyl ether)/water, Polymer 41 (2000) 3737-3743.

[56] M. Messori, M. Toselli, F. Pilati, E. Fabbri, P. Fabbri, L. Pasquali, S. Nannarone, Prevention of plasticizer leaching from PVC medical devices by using organic-inorganic hybrid coatings, Polymer 45 (2004) 805-813.

[57] N. Sarkar, Thermal gelation properties of methyl and hydroxypropyl methylcellulose, J. Appl. Polym. Sci. 24 (1979) 1073-1087.

[58] A. Carlsson, G. Karlström, B. Lindman, Thermal gelation of nonionic cellulose ethers and ionic surfactants in water, Colloids Surf. 47 (1990) 147-165.

[59] S.M.C. Silva, F.V. Pinto, F.E. Antunes, M.G. Miguel, J.J.S. Sousa, A.A.C.C. Pais, Aggregation and gelation in hydroxypropylmethyl cellulose aqueous solutions, J. Colloid Interface Sci. 327 (2008) 333-340.

[60] V.M.O. Cardoso, B.S.F. Cury, R.C. Evangelista, M.P.D. Gremião, Development and characterization of cross-linked gellan gum and retrograded starch blend hydrogels for drug delivery applications, J. Mech. Behav. Biomed. Mater. 65 (2017) 317-333.

[61] F.G. Prezotti, B.S.F. Cury, R.C. Evangelista, Mucoadhesive beads of gellan gum/ pectin intended to controlled delivery of drugs, Carbohydr. Polym. 113 (2014) 286-295.

[62] F.G. Prezotti, A.B. Meneguin, R.C. Evangelista, B.S.F. Cury, Preparation and characterization of free films of high amylose/pectin mixtures cross-linked with sodium trimetaphosphate, Drug Dev. Ind. Pharm. 38 (2012) 1354-1359.

[63] T. Osmałek, A. Froelich, S. Tasarek, Application of gellan gum in pharmacy and medicine, Int. J. Pharm. 466 (2014) 328-340.

[64] F.I. Boni, F.G. Prezotti, B.S.F. Cury, Gellan gum microspheres crosslinked with trivalent ion: Effect of polymer and crosslinker concentrations on drug release and mucoadhesive properties, Drug Dev. Ind. Pharm. 42 (2016) 1283-1290.

[65] J. Seuring, S. Agarwal, Polymers with upper critical solution temperature in aqueous solution: Unexpected properties from known building blocks, ACS Macro Lett. 2 (2013) 597-600.

[66] J. Seuring, S. Agarwal, Polymers with upper critical solution temperature in aqueous solution, Macromol. Rapid Commun. 33 (2012) 1898-1920.

[67] H. Tan, C.M. Ramirez, N. Miljkovic, H. Li, J.P. Rubin, K.G. Marra, Thermosensitive injectable hyaluronic acid hydrogel for adipose tissue engineering, Biomaterials 30 (2009) 6844-6853.

[68] H. Gao, W. Yang, K. Min, L. Zha, C. Wang, S. Fu, Thermosensitive poly(N-isopropylacrylamide) nanocapsules with controlled permeability, Polymer 46 (2005) 1087-1093.

[69] K.-F. Arndt, T. Schmidt, R. Reichelt, Thermo-sensitive poly(methyl vinyl ether) micro-gel formed by high energy radiation, Polymer 42 (2001) 6785-6791.

[70] O. Pillai, R. Panchagnula, Transdermal delivery of insulin from poloxamer gel: Ex vivo and in vivo skin permeation studies in rat using iontophoresis and chemica enhancers, J. Control. Release 89 (2003) 127-140.

[71] A. Chenite, C. Chaput, D. Wang, C. Combes, M.D. Buschmann, C.D. Hoemann, J.C. Leroux, B.L. Atkinson, F. Binette, A. Selmani, Novel injectable neutral solutions of chitosan form biodegradable gels in situ, Biomaterials 21 (2000) $2155-2161$.

[72] J. Hu, Y. Chen, Y. Li, Z. Zhou, Y. Cheng, A thermo-degradable hydrogel with lighttunable degradation and drug release, Biomaterials 112 (2017) 133-140.

[73] S.-H. Hu, T.-Y. Liu, D.-M. Liu, S.-Y. Chen, Nano-ferrosponges for controlled drug release, J. Control. Release 121 (2007) 181-189.

[74] P.M. Kharkar, K.L. Kiick, A.M. Kloxin, Design of thiol-and light-sensitive degradable hydrogels using Michael-type addition reactions, Polym. Chem. 6 (2015) $5565-5574$

[75] H.-W. Liu, S.-H. Hu, Y.-W. Chen, S.-Y. Chen, Characterization and drug release behavior of highly responsive chip-like electrically modulated reduced graphene oxide-poly (vinyl alcohol) membranes, J. Mater. Chem. 22 (2012) 17311-17320.

[76] T.-Y. Liu, S.-H. Hu, K.-H. Liu, D.-M. Liu, S.-Y. Chen, Preparation and characterization of smart magnetic hydrogels and its use for drug release, J. Magn. Magn. Mater. 304 (2006) e397-e399.

[77] T.-Y. Liu, S.-H. Hu, T.-Y. Liu, D.-M. Liu, S.-Y. Chen, Magnetic-sensitive behavior of intelligent ferrogels for controlled release of drug, Langmuir 22 (2006) 5974-5978.

[78] K. Sawahata, M. Hara, H. Yasunaga, Y. Osada, Electrically controlled drug delivery system using polyelectrolyte gels, J. Control. Release 14 (1990) 253-262.

[79] T.-S. Tsai, V. Pillay, Y.E. Choonara, L.C. Du Toit, G. Modi, D. Naidoo, P. Kumar, A polyvinyl alcohol-polyaniline based electro-conductive hydrogel for controlled stimuli-actuable release of indomethacin, Polymers 3 (2011) 150-172.

[80] X. Wang, C. Wang, Q. Zhang, Y. Cheng, Near infrared light-responsive and injectable supramolecular hydrogels for on-demand drug delivery, Chem. Commun. 52 (2016) 978-981.

[81] F.M. Andreopoulos, E.J. Beckman, A.J. Russell, Light-induced tailoring of PEGhydrogel properties, Biomaterials 19 (1998) 1343-1352.

[82] F.M. Andreopoulos, I. Persaud, Delivery of basic fibroblast growth factor (bFGF) rom photoresponsive hydrogel scaffolds, Biomaterials 27 (2006) 2468-2476.

[83] F.M. Andreopoulos, M.J. Roberts, M.D. Bentley, J.M. Harris, E.J. Beckman, A.J. Russell, Photoimmobilization of organophosphorus hydrolase within a PEGbased hydrogel, Biotechnol. Bioeng. 65 (1999) 579-588.

[84] S.J. Kim, S.J. Park, S.I. Kim, Swelling behavior of interpenetrating polymer network hydrogels composed of poly (vinyl alcohol) and chitosan, React. Funct. Polym. 55 (2003) 53-59.

[85] M.B. Mellott, K. Searcy, M.V. Pishko, Release of protein from highly cross-linked 
hydrogels of poly (ethylene glycol) diacrylate fabricated by UV polymerization, Biomaterials 22 (2001) 929-941.

[86] K.T. Nguyen, J.L. West, Photopolymerizable hydrogels for tissue engineering applications, Biomaterials 23 (2002) 4307-4314.

[87] L.S. Coons, B. Rangarajan, D. Godshall, A.B. Scranton, Photopolymerizations of vinyl ester: Glass fiber composites, in: A.B. Scranton, C.N. Bowman, R.W. Peiffer (Eds.), Photopolymerization, ACS Publications, 1997, pp. 203-218.

[88] K.M. Gattás-Asfura, E. Weisman, F.M. Andreopoulos, M. Micic, B. Muller, S. Sirpal, S.M. Pham, R.M. Leblanc, Nitrocinnamate-functionalized gelatin: Synthesis and "smart" hydrogel formation via photo-cross-linking, Biomacromolecules 6 (2005) 1503-1509.

[89] S.H. Kim, Y. Sun, J.A. Kaplan, M.W. Grinstaff, J.R. Parquette, Photo-crosslinking of a self-assembled coumarin-dipeptide hydrogel, New J. Chem. 39 (2015) 3225-3228.

[90] Y. Zheng, F.M. Andreopoulos, M. Micic, Q. Huo, S.M. Pham, R.M. Leblanc, A novel photoscissile poly (ethylene glycol)-based hydrogel, Adv. Funct. Mater. 11 (2001) $37-40$.

[91] B. Yan, J.-C. Boyer, D. Habault, N.R. Branda, Y. Zhao, Near infrared light triggered release of biomacromolecules from hydrogels loaded with upconversion nanoparticles, J. Am. Chem. Soc. 134 (2012) 16558-16561.

[92] F. Yanagawa, S. Sugiura, T. Takagi, K. Sumaru, G. Camci-Unal, A. Patel, A. Khademhosseini, T. Kanamori, Activated-ester-type photocleavable crosslinker for preparation of photodegradable hydrogels using a two-component mixing reaction, Adv. Healthc. Mater. 4 (2015) 246-254.

[93] K. Peng, I. Tomatsu, A. Kros, Light controlled protein release from a supramolecular hydrogel, Chem. Commun. 46 (2010) 4094-4096.

[94] G. Pouliquen, C. Tribet, Light-triggered association of bovine serum albumin and azobenzene-modified poly (acrylic acid) in dilute and semidilute solutions, Macromolecules 39 (2006) 373-383.

[95] G. Jalani, R. Naccache, D.H. Rosenzweig, L. Haglund, F. Vetrone, M. Cerruti, Photocleavable hydrogel-coated upconverting nanoparticles: A multifunctional theranostic platform for NIR imaging and on-demand macromolecular delivery, J. Am. Chem. Soc. 138 (2016) 1078-1083.

[96] K. Szaciłowski, W. Macyk, A. Drzewiecka-Matuszek, M. Brindell, G. Stochel, Bioinorganic photochemistry: Frontiers and mechanisms, Chem. Rev. 105 (2005) 2647-2694.

[97] F. Auzel, Upconversion and anti-stokes processes with $\mathrm{f}$ and $\mathrm{d}$ ions in solids, Chem. Rev. 104 (2004) 139-174.

[98] M. Haase, H. Schäfer, Upconverting nanoparticles, Angew. Chemie Int. Ed. 50 (2011) 5808-5829.

[99] I.C. Kwon, Y.H. Bae, T. Okano, S.W. Kim, Drug release from electric current sensitive polymers, J. Control. Release 17 (1991) 149-153 (156).

[100] R. Tomer, D. Dimitrijevic, A.T. Florence, Electrically controlled release of macromolecules from cross-linked hyaluronic acid hydrogels, J. Control. Release 33 (1995) 405-413.

[101] E.J. Choi, J. Shin, Z.H. Khaleel, I. Cha, S.-H. Yun, S.-W. Cho, C. Song, Synthesis of electroconductive hydrogel films by an electro-controlled click reaction and their application to drug delivery systems, Polym. Chem. 6 (2015) 4473-4478.

[102] S.H. Takahashi, L.M. Lira, S.I.C. de Torresi, Zero-order release profiles from a multistimuli responsive electro-conductive hydrogel, J. Biomater. Nanobiotechnol. 3 (2012) 262-268.

[103] J.L. Zhang, R.S. Srivastava, R.D. Misra, Core-shell magnetite nanoparticles surface encapsulated with smart stimuli-responsive polymer: Synthesis, characterization, and LCST of viable drug-targeting delivery system, Langmuir 23 (2007) 6342-6351.

[104] K. Hayashi, K. Ono, H. Suzuki, M. Sawada, M. Moriya, W. Sakamoto, T. Yogo, High-frequency, magnetic-field-responsive drug release from magnetic nanoparticle/organic hybrid based on hyperthermic effect, ACS Appl. Mater. Interfaces 2 (2010) 1903-1911.

[105] S.-H. Hu, T.-Y. Liu, D.-M. Liu, S.-Y. Chen, Controlled pulsatile drug release from a ferrogel by a high-frequency magnetic field, Macromolecules 40 (2007) 6786-6788.

[106] L. Zhou, B. He, F. Zhang, Facile one-pot synthesis of iron oxide nanoparticles crosslinked magnetic poly (vinyl alcohol) gel beads for drug delivery, ACS Appl. Mater. Interfaces 4 (2012) 192-199.

[107] B.-L. Guo, Q.-Y. Gao, Preparation and properties of a pH/temperature-responsive carboxymethyl chitosan/poly (N-isopropylacrylamide) semi-IPN hydrogel for oral delivery of drugs, Carbohydr. Res. 342 (2007) 2416-2422.

[108] D. Kuckling, A. Doering, F. Krahl, K.F. Arndt, Stimuli-responsive polymer systems, in: M. Moeller, K. Matyjaszewski (Eds.), Polymer Science: A Comprehensive Reference, Elsevier, Amsterdam, 2012, pp. 377-413.

[109] S.D. Horava, N.A. Peppas, Design of pH-responsive biomaterials to enable the oral route of hematological factor IX, Ann. Biomed. Eng. 44 (2016) 1970-1982.

[110] M. Constantin, S. Bucatariu, V. Harabagiu, I. Popescu, P. Ascenzi, G. Fundueanu, Poly (N-isopropylacrylamide-co-methacrylic acid) $\mathrm{pH} /$ thermo-responsive porous hydrogels as self-regulated drug delivery system, Eur. J. Pharm. Sci. 62 (2014) 86-95.

[111] M. Caldorera-Moore, K. Maass, R. Hegab, G. Fletcher, N. Peppas, Hybrid responsive hydrogel carriers for oral delivery of low molecular weight therapeutic agents, J. Drug Deliv. Sci. Technol. 30 (2015) 352-359.

[112] E.S. Gil, S.M. Hudson, Stimuli-reponsive polymers and their bioconjugates, Prog. Polym. Sci. 29 (2004) 1173-1222.

[113] S. Sanjuan, P. Perrin, N. Pantoustier, Y. Tran, Synthesis and swelling behavior of pH-responsive polybase brushes, Langmuir 23 (2007) 5769-5778.

[114] N. Cohen, L. Binyamin, Y. Levi-Kalisman, G.Y. Berguig, A. Convertine, P. Stayton, R. Yerushalmi-Rozen, $\mathrm{pH}$ and salt effects on surface activity and self-assembly of copolymers containing a weak polybase, Langmuir 32 (2016) 9286-9292.

[115] S.S. Vaghani, M.M. Patel, C.S. Satish, K.M. Patel, N.P. Jivani, Synthesis and characterization of carboxymethyl chitosan hydrogel: Application as $\mathrm{pH}$-sensitive delivery for nateglinide, Curr. Drug Deliv. 9 (2012) 628-636.

[116] S. Maya, B. Sarmento, A. Nair, N.S. Rejinold, S.V. Nair, R. Jayakumar, Smart stimuli sensitive nanogels in cancer drug delivery and imaging: A review, Curr. Pharm. Des. 19 (2013) 7203-7218.

[117] H.-Y. Park, I.-H. Song, J.-H. Kim, W.-S. Kim, Preparation of thermally denatured albumin gel and its pH-sensitive swelling, Int. J. Pharm. 175 (1998) 231-236.

[118] A.W. Chan, R.J. Neufeld, Modeling the controllable pH-responsive swelling and pore size of networked alginate based biomaterials, Biomaterials 30 (2009) 6119-6129.

[119] J. Guo, G. Kaletunç, Dissolution kinetics of pH responsive alginate-pectin hydrogel particles, Food Res. Int. 88 (2016) 129-139.

[120] C. Liu, E. Thormann, P.M. Claesson, E. Tyrode, Surface grafted chitosan gels. Part II. Gel formation and characterization, Langmuir 30 (2014) 8878-8888.

[121] X. Qu, A. Wirsen, A.-C. Albertsson, Novel pH-sensitive chitosan hydrogels: Swelling behavior and states of water, Polymer 41 (2000) 4589-4598.

[122] J. Yang, J. Chen, D. Pan, Y. Wan, Z. Wang, pH-sensitive interpenetrating network hydrogels based on chitosan derivatives and alginate for oral drug delivery, Carbohydr. Polym. 92 (2013) 719-725.

[123] K.A. Watkins, R. Chen, pH-responsive, lysine-based hydrogels for the oral delivery of a wide size range of molecules, Int. J. Pharm. 478 (2015) 496-503.

[124] X. Gao, C. He, C. Xiao, X. Zhuang, X. Chen, Biodegradable pH-responsive polyacrylic acid derivative hydrogels with tunable swelling behavior for oral delivery of insulin, Polymer 54 (2013) 1786-1793.

[125] D. Buenger, F. Topuz, J. Groll, Hydrogels in sensing applications, Prog. Polym. Sci. 37 (2012) 1678-1719.

[126] T.G. Park, A.S. Hoffman, Sodium chloride-induced phase transition in nonionic poly (N-isopropylacrylamide) gel, Macromolecules 26 (1993) 5045-5048.

[127] F. Horkay, I. Tasaki, P.J. Basser, Osmotic swelling of polyacrylate hydrogels in physiological salt solutions, Biomacromolecules 1 (2000) 84-90.

[128] S.G. Roy, A. Kumar, P. De, Amino acid containing cross-linked co-polymer gels: pH, thermo and salt responsiveness, Polymer 85 (2016) 1-9.

[129] Y. Zhao, H. Su, L. Fang, T. Tan, Superabsorbent hydrogels from poly (aspartic acid) with salt-, temperature-and pH-responsiveness properties, Polymer 46 (2005) 5368-5376.

[130] X.-W. Liu, S. Zhu, S.-R. Wu, P. Wang, G.-Z. Han, Response behavior of ion-sensitive hydrogel based on crown ether, Colloids Surfaces A Physicochem. Eng. Asp. 417 (2013) 140-145.

[131] A. Sgambato, L. Cipolla, L. Russo, Bioresponsive hydrogels: Chemical strategies and perspectives in tissue engineering, Gels 2 (2016) 28-41.

[132] M.F. Maitz, U. Freudenberg, M.V. Tsurkan, M. Fischer, T. Beyrich, C. Werner, Bioresponsive polymer hydrogels homeostatically regulate blood coagulation, Nat. Commun. 4 (2013) 2168.

[133] D. Roy, J.N. Cambre, B.S. Sumerlin, Future perspectives and recent advances in stimuli-responsive materials, Prog. Polym. Sci. 35 (2010) 278-301.

[134] T. Miyata, T. Uragami, K. Nakamae, Biomolecule-sensitive hydrogels, Adv. Drug Deliv. Rev. 54 (2002) 79-98.

[135] T. Miyata, Biomolecule-responsive hydrogels, in: R.M. Ottenbrite, K. Park, T. Okano (Eds.), Biomedical Applications of Hydrogels Handbook, SpringerVerlag, New York, 2010, pp. 65-86.

[136] A. Kawamura, T. Miyata, Biologically stimuli-responsive hydrogels, in: Q. Li (Ed.), Intelligent Stimuli-Responsive Materials: From Well-Defined Nanostructures to Applications, John Wiley \& Sons, New Jersey, 2013, pp. 335-362.

[137] E. Holowka, S.K. Bhatia, Drug Delivery: Materials Design and Clinical Perspective, Springer-Verlag, New York, 2014.

[138] Y. Bae, S. Fukushima, A. Harada, K. Kataoka, Design of environment-sensitive supramolecular assemblies for intracellular drug delivery: Polymeric micelles that are responsive to intracellular pH change, Angew. Chemie. 42 (2003) 4640-4643.

[139] M.E. Caldorera-Moore, W.B. Liechty, N.A. Peppas, Responsive theranostic systems: Integration of diagnostic imaging agents and responsive controlled release drug delivery carriers, Acc. Chem. Res. 44 (2011) 1061-1070.

[140] P.P. Kalshetti, V.B. Rajendra, D.N. Dixit, P.P. Parekh, Hydrogels as a drug delivery system and applications: A review, Int. J. Pharm. Pharm. Sci. 4 (2012) 1-7.

[141] J. Kopeček, Smart and genetically engineered biomaterials and drug delivery systems, Eur. J. Pharm. Sci. 20 (2003) 1-16.

[142] S. Mura, J. Nicolas, P. Couvreur, Stimuli-responsive nanocarriers for drug delivery, Nat. Mater. 12 (2013) 991-1003.

[143] G. Albin, T.A. Horbett, B.D. Ratner, Glucose sensitive membranes for controlled delivery of insulin: Insulin transport studies, J. Control. Release 2 (1985) 153-164.

[144] Y. Dong, W. Wang, O. Veiseh, E.A. Appel, K. Xue, M.J. Webber, B.C. Tang, X.W. Yang, G.C. Weir, R. Langer, D.G. Anderson, Injectable and glucose-responsive hydrogels based on boronic acid-glucose complexation, Langmuir 32 (2016) 8743-8747.

[145] T. Traitel, Y. Cohen, J. Kost, Characterization of glucose-sensitive insulin release systems in simulated in vivo conditions, Biomaterials 21 (2000) 1679-1687.

[146] R.V. Ulijn, Enzyme-responsive materials: A new class of smart biomaterials, J. Mater. Chem. 16 (2006) 2217-2225.

[147] T. Miyata, N. Asami, T. Uragami, A reversibly antigen-responsive hydrogel, Nature 399 (1999) 766-769.

[148] Z.-R. Lu, P. Kopečková, J. Kopeček, Antigen responsive hydrogels based on polymerizable antibody Fab' fragment, Macromol. Biosci. 3 (2003) 296-300.

[149] R. Zhang, A. Bowyer, R. Eisenthal, J. Hubble, A smart membrane based on an antigen-responsive hydrogel, Biotechnol. Bioeng. 97 (2007) 976-984.

[150] M. Ikeda, R. Ochi, A. Wada, I. Hamachi, Supramolecular hydrogel capsule showing 
prostate specific antigen-responsive function for sensing and targeting prostate cancer cells, Chem. Sci. 1 (2010) 491-498.

[151] A. Kumar, A. Srivastava, I.Y. Galaev, B. Mattiasson, Smart polymers: Physical forms and bioengineering applications, Prog. Polym. Sci. 32 (2007) 1205-1237.

[152] H.P. James, R. John, A. Alex, K.R. Anoop, Smart polymers for the controlled delivery of drugs-a concise overview, Acta Pharm. Sin. B 4 (2014) 120-127.

[153] P. Bernfeld, J. Wan, Antigens and enzymes made insoluble by entrapping them into lattices of synthetic polymers, Science 142 (1963) 678-679.

[154] Y. Takemoto, H. Ajiro, T.-A. Asoh, M. Akashi, Fabrication of surface-modified hydrogels with polyion complex for controlled release, Chem. Mater. 22 (2010) 2923-2929.

[155] J.P. Lorand, J.O. Edwards, Polyol complexes and structure of the benzeneboronate ion, J. Org. Chem. 24 (1959) 769-774.

[156] R.V. Ulijn, N. Bibi, V. Jayawarna, P.D. Thornton, S.J. Todd, R.J. Mart, A.M. Smith, J.E. Gough, Bioresponsive hydrogels, Mater. Today 10 (2007) 40-48.

[157] T. McDonald, A. Patrick, R. Williams, B.G. Cousins, R.V. Ulijn, Bio-responsive hydrogels for biomedical applications, in: F. Carpi, E. Smela (Eds.), Biomedical Applications of Electroactive Polymer Actuators, John Wiley \& Sons, Chichester, UK, 2009, pp. 43-59.

[158] Y. Park, M.P. Lutolf, J.A. Hubbell, E.B. Hunziker, M. Wong, Bovine primary chondrocyte culture in synthetic matrix metalloproteinase-sensitive poly (ethylene glycol)-based hydrogels as a scaffold for cartilage repair, Tissue Eng. 10 (2004) 515-522.

[159] C.S. Satish, K.P. Satish, H.G. Shivakumar, Hydrogels as controlled drug delivery systems: Synthesis, crosslinking, water and drug transport mechanism, Indian, J. Pharm. Sci. 68 (2006) 133-140.

[160] L. Hovgaard, H. Brøndsted, Dextran hydrogels for colon-specific drug delivery, J. Control. Release 36 (1995) 159-166.

[161] A. Jain, Y. Gupta, S.K. Jain, Perspectives of biodegradable natural polysaccharides for site-specific drug delivery to the colon, J Pharm Pharm Sci. 10 (2007) 86-128.

[162] H.L. Lim, Y. Hwang, M. Kar, S. Varghese, Smart hydrogels as functional biomimetic systems, Biomater. Sci. 2 (2014) 603-618.

[163] A.E. Nel, L. Mädler, D. Velegol, T. Xia, E.M.V. Hoek, P. Somasundaran, F. Klaessig, V. Castranova, M. Thompson, Understanding biophysicochemical interactions at the nano-bio interface, Nat. Mater. 8 (2009) 543-557.

[164] M. Liu, H. Du, Z. Wenjia, G. Zhai, Internal stimuli-responsive nanocarriers for drug delivery: Design strategies and applications, Mater. Sci. Eng., C 71 (2017) 1267-1280.

[165] R. Medzhitov, Inflammation 2010: New adventures of an old flame, Cell 140 (2010) (2010) 771-776.

[166] V. Deretic, Links between autophagy, innate immunity, inflammation and Crohn's disease, Dig. Dis. 27 (2009) 246-251.

[167] M.P. Lutolf, J.L. Lauer-Fields, H.G. Schmoekel, A.T. Metters, F.E. Weber, G.B. Fields, J.A. Hubbell, Synthetic matrix metalloproteinase-sensitive hydrogels for the conduction of tissue regeneration: Engineering cell-invasion characteristics, Proc. Natl. Acad. Sci. 100 (2003) 5413-5418.

[168] C. Gabay, A. So, Redefining treatment of rheumatic arthritis with anti-cytokines, Rev. Med Suisse 3 (2007) 715-717.

[169] N. Butoescu, O. Jordan, E. Doelker, Intra-articular drug delivery systems for the treatment of rheumatic diseases: A review of the factors influencing their perfor mance, Eur. J. Pharm. Biopharm. 73 (2009) 205-218.

[170] F.R. Fernandez, C.B. Grindem, A.J. Lipowitz, V. Perman, Synovial fluid analysis: Preparation of smears for cytologic examination of canine synovial fluid, J. Am. Anim. Hosp. Assoc. 19 (1983) 727-734.

[171] L. Punzi, F. Oliviero, Arthrocentesis and synovial fluid analysis in clinical practice, Ann. N. Y. Acad. Sci. 1154 (2009) 152-158.

[172] D. Song, B. Liu, P. Li, L.Q. Bi, Evaluation of MRI in the diagnosis of rheumatoid arthritis, Zhonghua Yi Xue Za Zhi 90 (2010) 3049-3053.

[173] A.A. Aimetti, A.J. Machen, K.S. Anseth, Poly (ethylene glycol) hydrogels formed by thiol-ene photopolymerization for enzyme-responsive protein delivery, Biomaterials 30 (2009) 6048-6054.

[174] S. Toledano, R.J. Williams, V. Jayawarna, R.V. Ulijn, Enzyme-triggered self-assembly of peptide hydrogels via reversed hydrolysis, J. Am. Chem. Soc. 128 (2006) 1070-1071.

[175] J.L. Drury, D.J. Mooney, Hydrogels for tissue engineering: Scaffold design variables and applications, Biomaterials 24 (2003) 4337-4351.

[176] D.K. Podolsky, Inflammatory bowel disease, N. Engl. J. Med. 347 (2002) 417-429.

[177] B. Tirosh, N. Khatib, Y. Barenholz, A. Nissan, A. Rubinstein, Transferrin as a luminal target for negatively charged liposomes in the inflamed colonic mucosa, Mol. Pharm. 6 (2009) 1083-1091.

[178] L. Sorokin, The impact of the extracellular matrix on inflammation, Nat. Rev. Immunol. 10 (2010) 712-723.

[179] S. Zhang, J. Ermann, M.D. Succi, A. Zhou, M.J. Hamilton, B. Cao, J.R. Korzenik, J.N. Glickman, P.K. Vemula, L.H. Glimcher, G. Traverso, R. Langer, J.M. Karp, An inflammation-targeting hydrogel for local drug delivery in inflammatory bowel disease, Sci. Transl. Med. 7 (2015) 300ra128.

[180] J. Zhou, M. O'Keeffe, G. Liao, F. Zhao, C. Terhorst, B. Xu, Design and synthesis of nanofibers of self-assembled de novo glycoconjugates towards mucosal lining restoration and anti-inflammatory drug delivery, Tetrahedron 72 (2016) 6078-6083.

[181] T.G. Burish, D.M. Tope, Psychological techniques for controlling the adverse side effects of cancer chemotherapy: Findings from a decade of research, J. Pain Symptom Manage. 7 (1992) 287-301.

[182] I.F. Tannock, D. Rotin, Acid pH in tumors and its potential for therapeutic ex ploitation, Cancer Res. 49 (1989) 4373-4384.

[183] J.-W. Choi, S.-J. Jung, D. Kasala, J.K. Hwang, J. Hu, Y.H. Bae, C.-O. Yun, pH- sensitive oncolytic adenovirus hybrid targeting acidic tumor microenvironment and angiogenesis, J. Control. Release 205 (2015) 134-143.

[184] M. Curtarello, E. Zulato, G. Nardo, S. Valtorta, G. Guzzo, E. Rossi, G. Esposito, A. Msaki, A. Pastò, A. Rasola, L. Persano, F. Ciccarese, R. Bertorelle, S. Todde, M. Plebani, H. Schroer, S. Walenta, W. Mueller-Klieser, A. Amadori, R.M. Moresco, S. Indraccolo, VEGF-targeted therapy stably modulates the glycolytic phenotype of tumor cells, Cancer Res. 75 (2015) 120-133.

[185] O. Warburg, On the origin of cancer cells, Science 123 (1956) 309-314.

[186] C. Pinheiro, A. Longatto-Filho, J. Azevedo-Silva, M. Casal, F.C. Schmitt, F. Baltazar, Role of monocarboxylate transporters in human cancers: State of the art, J. Bioenerg. Biomembr. 44 (2012) 127-139.

[187] V. Miranda-Gonçalves, M. Honavar, C. Pinheiro, O. Martinho, M.M. Pires, C. Pinheiro, M. Cordeiro, G. Bebiano, P. Costa, I. Palmeirim, R.M. Reis, F. Baltazar, Monocarboxylate transporters (MCTs) in gliomas: Expression and exploitation as therapeutic targets, Neuro. Oncol. 15 (2013) 172-188.

[188] L. Tian, Y.H. Bae, Cancer nanomedicines targeting tumor extracellular pH, Colloids Surfaces B Biointerfaces 99 (2012) 116-126.

[189] A.K. Mitra, V. Agrahari, A. Mandal, K. Cholkar, C. Natarajan, S. Shah, M. Joseph, H.M. Trinh, R. Vaishya, X. Yang, Y. Hao, V. Khurana, D. Pal, Novel delivery approaches for cancer therapeutics, J. Control. Release 219 (2015) 248-268.

[190] N.N. Ferreira, L.M.B. Ferreira, V. Miranda-Gonçalves, R.M. Reis, T.V. Seraphim, J.C. Borges, F. Baltazar, M.P.D. Gremião, Alginate hydrogel improves anti-angiogenic bevacizumab activity in cancer therapy, Eur. J. Pharm. Biopharm. 119 (2017) 271-282.

[191] L. Li, J. Gu, J. Zhang, Z. Xie, Y. Lu, L. Shen, Q. Dong, Y. Wang, Injectable and biodegradable $\mathrm{pH}$-responsive hydrogels for localized and sustained treatment of human fibrosarcoma, ACS Appl. Mater. Interfaces 7 (2015) 8033-8040.

[192] X. Chen, Z. Liu, A pH-responsive hydrogel based on a tumor-targeting mesoporous silica nanocomposite for sustained cancer labeling and therapy, Macromol. Rapid Commun. 37 (2016) 1533-1539.

[193] T.K. Giri, Alginate Containing Nanoarchitectonics for Improved Cancer Therapy, in: A.M. Holban, A. Grumezescu (Eds.), Nanoarchitectonics for Smart Delivery and Drug Targeting, first ed., William Andrew, 2016.

[194] N.N. Ferreira, T.A. Perez, L.N. Pedreiro, F.G. Prezotti, F.I. Boni, V.M.O. Cardoso, T. Venâncio, M.P.D. Gremião, A novel pH-responsive hydrogel-based on calcium alginate engineered by the previous formation of polyelectrolyte complexes (PECs) intended to vaginal administration, Drug Dev. Ind. Pharm. 43 (2017) 1656-1668.

[195] K. Na, E.S. Lee, Y.H. Bae, Self-organized nanogels responding to tumor extracellular pH: pH-dependent drug release and in vitro cytotoxicity against MCF-7 cells, Bioconjug. Chem. 18 (2007) 1568-1574.

[196] K. Raemdonck, J. Demeester, S. De Smedt, Advanced nanogel engineering for drug delivery, Soft Matter 5 (2009) 707-715.

[197] A.K. Samadi, A.G. Georgakilas, A. Amedei, A. Amin, A. Bishayee, B.L. Lokeshwar, B. Grue, C. Panis, C.S. Boosani, D. Poudyal, D.M. Stafforini, D. Bhakta, E. Niccolai, G. Guha, H.P.V. Rupasinghe, H. Fujii, K. Honoki, K. Mehta, K. Aquilano, L. Lowe, L.J. Hofseth, L. Ricciardiello, M.R. Ciriolo, N. Singh, R.L. Whelan, R. Chaturvedi, S.S. Ashraf, H.S. Kumara, S. Nowsheen, S.I. Mohammed, W.G. Helferich, X. Yang, A multi-targeted approach to suppress tumor-promoting inflammation, Semin. Cancer Biol. 35 (2015) S151-S184.

[198] Y. Wang, H. Xu, J. Wang, L. Ge, J. Zhu, Development of a thermally responsive nanogel based on chitosan-poly ( $\mathrm{N}$-isopropylacrylamide-co-acrylamide) for paclitaxel delivery, J. Pharm. Sci. 103 (2014) 2012-2021.

[199] X. Zhu, Y. Zhang, H. Huang, H. Zhang, L. Hou, Z. Zhang, Functionalized graphene oxide-based thermosensitive hydrogel for near-infrared chemo-photothermal therapy on tumor, J. Biomater. Appl. 30 (2016) 1230-1241.

[200] L.E. Strong, S.N. Dahotre, J.L. West, Hydrogel-nanoparticle composites for optically modulated cancer therapeutic delivery, J. Control. Release 178 (2014) $63-68$.

[201] A. Basu, K.R. Kunduru, S. Doppalapudi, A.J. Domb, W. Khan, Poly (lactic acid) based hydrogels, Adv. Drug Deliv. Rev. 107 (2016) 192-205.

[202] Z. Wu, X. Zou, L. Yang, S. Lin, J. Fan, B. Yang, X. Sun, Q. Wan, Y. Chen, S. Fu, Thermosensitive hydrogel used in dual drug delivery system with paclitaxelloaded micelles for in situ treatment of lung cancer, Colloids Surfaces B Biointerfaces 122 (2014) 90-98.

[203] Y. Chau, Y. Luo, A.C.Y. Cheung, Y. Nagai, S. Zhang, J.B. Kobler, S.M. Zeitels, R. Langer, Incorporation of a matrix metalloproteinase-sensitive substrate into self-assembling peptides-a model for biofunctional scaffolds, Biomaterials 29 (2008) 1713-1719.

[204] L. Nakopoulou, I. Tsirmpa, P. Alexandrou, A. Louvrou, C. Ampela, S. Markaki, P.S. Davaris, MMP-2 protein in invasive breast cancer and the impact of MMP-2/ TIMP-2 phenotype on overall survival, Breast Cancer Res. Treat. 77 (2003) $145-155$.

[205] R. Price, A. Poursaid, J. Cappello, H. Ghandehari, In vivo evaluation of matrix metalloproteinase responsive silk-elastinlike protein polymers for cancer gene therapy, J. Control. Release 213 (2015) 96-102.

[206] V.K. Garripelli, J.-K. Kim, S. Son, W.J. Kim, M.A. Repka, S. Jo, Matrix metalloproteinase-sensitive thermogelling polymer for bioresponsive local drug delivery, Acta Biomater. 7 (2011) 1984-1992.

[207] S. Wild, G. Roglic, A. Green, R. Sicree, H. King, Global prevalence of diabetes: Estimates for the year 2000 and projections for 2030, Diabetes Care 27 (2004) 1047-1053.

[208] N. Yadav, G. Morris, S.E. Harding, S. Ang, G.G. Adams, Various non-injectable delivery systems for the treatment of diabetes mellitus, Endocr. Metab. Immune Disord. Drug Targets 9 (2009) 1-13.

[209] J.C. Ozougwu, K.C. Obimba, C.D. Belonwu, C.B. Unakalamba, The pathogenesis and pathophysiology of type 1 and type 2 diabetes mellitus, J. Physiol. 
Pathophysiol. 4 (2013) 46-57.

[210] K.M. Gillespie, Type 1 diabetes: Pathogenesis and prevention, Can. Med. Assoc. J. 175 (2006) 165-170.

211] R.A. DeFronzo, D. Tripathy, Skeletal muscle insulin resistance is the primary defect in type 2 diabetes, Diabetes Care 32 (2009) S157-S163.

[212] R.I.G. Holt, Diagnosis, epidemiology and pathogenesis of diabetes mellitus: An update for psychiatrists, Br. J. Psych. 184 (2004) s55-s63.

[213] M.J. Webber, D.G. Anderson, Smart approaches to glucose-responsive drug delivery, J. Drug Target. 23 (2015) 651-655:

[214] R. Merindol, A. Walther, Materials learning from life: Concepts for active, adaptive and autonomous molecular systems, Chem. Soc. Rev. 46 (2017) 5588-5619. 